\title{
CONTROLLER DESIGN FOR BUSH-TYPE 1-D WAVE NETWORKS*
}

\author{
YAXUAN ZHANG ${ }^{1}$ AND GENQI XU ${ }^{1}$
}

\begin{abstract}
In this paper, we introduce a new method for feedback controller design for the complex distributed parameter networks governed by wave equations, which ensures the stability of the closed loop system. This method is based on the uniqueness theory of ordinary differential equations and cutting-edge approach in the graph theory, but it is not a simple extension. As a realization of this idea, we investigate a bush-type wave network. The well-posedness of the closed loop system is obtained via Lax-Milgram's lemma and semigroup theory. The validity of cutting-edge method is proved by spectral analysis approach. In particular, we give a detailed procedure of cutting-edge for the bush-type wave networks. The results show that if we impose feedback controllers, consisting of velocity and position terms, at all the boundary vertices and at most three velocity feedback controllers on the cycle, the system is asymptotically stabilized. Finally, some examples are given.
\end{abstract}

Mathematics Subject Classification. 35L05, 35L90, 37L15, 93C20, 93D15.

Received March 16, 2010. Revised July 31, 2010.

Published online December 2, 2010.

\section{INTRODUCTION}

Many real-world situations can conveniently be described by 1-D multi-link flexible structures (distributed parameter networks), including wave networks and beam networks. The study on these distributed parameter networks has caught much attention in mathematics and engineering since 80's last century. Many nice results are available, covering the controllability, observability and stabilization of the networks (e.g., $[1,3,7,9,11,13,14$, $17,19]$ ). Most of them are concerned with 1-D wave and beam equation expanded on simple graphs, while a few focus on the complex networks which have a number of edges and complicated connections. For example, the stabilization of a circular string was considered recently in [10] and a feedback law (with delay) acting at a single position of the cycle was presented to guarantee the exponential energy decay.

For the complex networks, imposing controllers at every vertex is not realistic, because it will result in high cost in engineering and complicated stability analysis in mathematics. The questions are: can we use fewer controllers to stabilize the system? If the answer is yes, how many controllers are needed at least and where should they be set up (this problem is itself an optimal one)? If the controllers have been designed, how can we test the stability of the corresponding controlled network in a simple way? These are challenging problems in both mathematics and engineering.

Keywords and phrases. Bush-type, wave network, controller design, asymptotic stability, cutting-edge.

* This research is supported by the Natural Science Foundation of China grant NSFC-60874034 and the Seed Foundation of Tianjin University.

1 Department of Mathematics, Tianjin University, Tianjin 300072, P.R. China. bunnyxuan@tju.edu.cn; gqxu@tju.edu.cn 
In the present paper we consider the controller design and stability analysis for complex networks. Here we introduce a new idea called "cutting-edge" method, by which we can get a control strategy, and at the same time it also ensures the asymptotic stability of the closed loop system. Our method is based on the following result.

Theorem 1.1. Let $X$ be a Hilbert space, and let $A$ be a compact resolvent operator which generates a $C_{0}$ semigroup $T(t)$ of contraction on $X$. Then there exist two invariant subspaces $X_{1}$ and $X_{2}$ of $T(t)$ such that $X=X_{1} \oplus X_{2}$, where

and

$$
X_{1}=\left\{x \in X \mid \lim _{t \rightarrow \infty} T(t) x=\lim _{t \rightarrow \infty} T^{*}(t) x=0\right\}
$$

$$
X_{2}=\left\{x \in X \mid\|T(t) x\|=\left\|T^{*}(t) x\right\|=\|x\|, \forall t \geq 0\right\} .
$$

Note that $X_{1}$ corresponds to the stable subspace of $X$, and that $X_{2}$ corresponds to the unstable subspace. So a direct idea is to divide a complex network into some subnetworks, and then to design controllers for each subnetwork so that the corresponding system is stable; and finally to combine the results appropriately. This procedure is complete via the following steps:

Step 1. Impose controllers at all the boundary vertices;

Step 2. "Cut" some edges from the graph according to the decomposition of the dissipative semigroup associated with the system. Here we hope the rest of the graph after "cutting-edge" is a subgraph;

Step 3. If the subgraph obtained in Step 2 is a null graph, the controller design is over; otherwise, impose suitable controllers on the subgraph, then go to Step 2, until all the edges of the original graph are "cut".

When all the edges are "cut", the system should be at least asymptotically stable, and all the controllers imposed to the system in this process form the desired control strategy.

In this process, the most important is Step 3, where we need to impose appropriate controllers according to the connection manner of the network. To illustrate this, we give a counterexample as follows:

$$
\left\{\begin{array}{l}
w_{t t}(x, t)=w_{x x}(x, t), \quad x \in(0,1) \\
w(0, t)=0, \quad w_{x}(1, t)=-\alpha w_{t}(1, t), \quad \alpha>0 \\
w(x, 0)=w_{0}(x), \quad w_{t}(x, 0)=w_{1}(x)
\end{array}\right.
$$

and

$$
\left\{\begin{array}{l}
w_{t t}(x, t)=w_{x x}(x, t), \quad x \in(1,2) \\
w(2, t)=0, \quad w_{x}(1, t)=\alpha w_{t}(1, t), \quad \alpha>0 \\
w(x, 0)=\widehat{w}_{0}(x), \quad w_{t}(x, 0)=\widehat{w}_{1}(x) .
\end{array}\right.
$$

Obviously, the systems (1.1) and (1.2) are exponentially stable. But the composite system

$$
\left\{\begin{array}{l}
w_{t t}(x, t)=w_{x x}(x, t), \quad x \in(0,1) \cup(1,2) \\
w(0, t)=0, \quad w(2, t)=0, \quad w\left(1^{+}, t\right)=w\left(1^{-}, t\right) \\
w_{x}\left(1^{-}, t\right)-w_{x}\left(1^{+}, t\right)=-\alpha w_{t}(1, t), \quad \alpha>0 \\
w(x, 0)=\bar{w}_{0}(x), \quad w_{t}(x, 0)=\bar{w}_{1}(x)
\end{array}\right.
$$

is unstable.

The "cutting-edge" approach is merely an idea for controller design. In the present paper we shall use this approach to design feedback controllers for 1-D wave system defined on bush-type graph, which is an arbitrary graph that has exactly one cycle and some trees rooted at the vertices of the cycle, shown as in Figure 1 for instance. The controller design is carried out from the boundary of the trees to the cycle. According to the existing results, for instance, see $[2,4,6,8,15,20]$, we can prove that a tree-shaped wave network is asymptotically stabilized if every boundary vertex is equipped with a velocity feedback controller. Therefore, imposing controllers at all the boundary vertices of a bush-type wave network will "cut" all the trees; the subgraph obtained after "cutting-edge" is only the cycle, of which the displacements at vertices across the trees are 0 . 


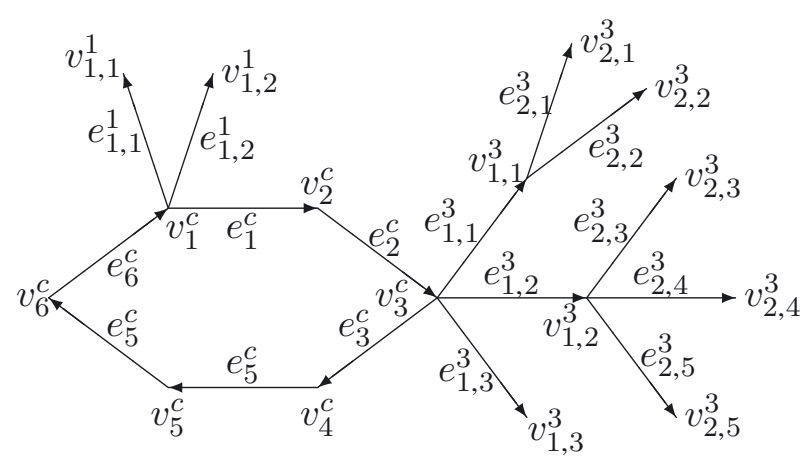

Figure 1. An example of bush-type graphs.

Now we design at most three controllers at appropriate positions so that the whole cycle can be "cut", and hence the bush-type wave network is asymptotically stable.

The rest is organized as follows. In Section 2, we present the description of the bush-type wave network, and the solvability of the system via Lax-Milgram's lemma and semigroup theory in an appropriate state space. The main result is given in Section 3, in which the number and locations of the controllers are provided. In addition, the validity of "cutting-edge" method, which ensures the stability, is proved for bush-type wave network by the spectral analysis [16]. In Section 4 we give some examples to show that for a concrete graph, we can use fewer controllers to stabilize the system. Finally, in Section 5, we conclude the results of the present paper.

\section{Model DescRiption AND SOlVABILITy OF THE ClOSED LOOP SYSTEM}

In this section, we describe the bush-type wave network under consideration and prove its solvability under more general assumptions.

\subsection{Bush-type wave network}

Let $\mathcal{G}=\mathcal{C} \cup \mathcal{T}$ be a bush-type graph with vertex set $V$ and edge set $E$, where $\mathcal{C}$ is a cycle with vertex set $V_{c}=\left\{v_{1}^{c}, v_{2}^{c}, \ldots, v_{N_{c}}^{c}\right\}$ and edge set $E_{c}=\left\{e_{1}^{c}, e_{2}^{c}, \ldots, e_{N_{c}}^{c}\right\}$, whose index set is denoted by $I_{c}=\left\{1,2, \ldots, N_{c}\right\}$, and $\mathcal{T}$ is a tree set whose roots are vertices of $\mathcal{C}$. Let $I_{T} \subset I_{c}$ be the index set of the roots of the trees in $\mathcal{T}$, and $\mathcal{T}_{k}$ be the tree rooted at vertex $v_{k}^{c}, k \in I_{T}$. Then $\mathcal{T}=\cup_{k \in I_{T}} \mathcal{I}_{k}$.

For a tree $\mathcal{T}_{k}, k \in I_{T}$, and two points $u_{1}, u_{2} \in \mathcal{T}_{k}$, if $u_{1}$ lies on a path that connects the root $v_{k}^{c}$ with $u_{2}$, we write $u_{1} \leq u_{2}$. Set $\left[u_{1}, u_{2}\right]=\left\{z \in \mathcal{T}_{k} \mid u_{1} \leq z \leq u_{2}\right\}$. If an edge $e=[v, \widehat{v}]$, then $v$ is called the start point of $e$ and $\widehat{v}$ the end point. For each vertex $v \in \mathcal{T}_{k}$, denote by $|v|$ the number of the edges between $v_{k}^{c}$ and $v$, which is called the order of $v$. For each edge $e \in \mathcal{T}_{k}$, its order is defined as the order of its end point. The number $p_{k}:=\max \left\{|v| \mid v \in \mathcal{T}_{k}\right\}$ is called the height of $\mathcal{T}_{k}$. Then the vertex set and edge set of $\mathcal{T}_{k}$ can be written as

$$
V_{k}=\left\{v_{k}^{c}, v_{j, i}^{k} \mid j=1,2, \ldots, p_{k}, i=1,2, \ldots, N_{k, j}\right\}, \quad E_{k}=\left\{e_{j, i}^{k} \mid j=1,2, \ldots, p_{k}, i=1,2, \ldots, N_{k, j}\right\}
$$

where $j$ is the order and $N_{k, j}$ is the number of $j$-order vertices (hence the edges) in $\mathcal{T}_{k}$.

For clarity, we arrange the elements of $V_{k}$ and $E_{k}$ in the increasing order of $j$ and $i, e . g$.,

$$
V_{k}=\left\{v_{k}^{c}, v_{1,1}^{k}, v_{1,2}^{k}, \ldots, v_{1, N_{k, 1}}^{k}, v_{2,1}^{k}, v_{2,2}^{k}, \ldots, v_{2, N_{k, 2}}^{k}, \ldots, v_{p_{k}, 1}^{k}, v_{p_{k}, 2}^{k}, \ldots, v_{p_{k}, N_{k, p_{k}}}^{k}\right\} .
$$

Let $N_{k}$ denote the number of the edges of $\mathcal{T}_{k}$, then $N_{k}>0$ and $N:=\sum_{k \in I_{T}} N_{k}+N_{c}$ is the number of the edges (hence the vertices) of $\mathcal{G}$. 
To simplify the description, for $k \in I_{c}$, we view $k+1$ as 1 if $k=N_{c}$ and $k-1$ as $N_{c}$ if $k=1$. For $e_{k}^{c}, k \in I_{c}$, we always denote by $v_{k}^{c}$ its start point and $v_{k+1}^{c}$ its end point. The direction of edge $e \in \mathcal{G}$ is defined from its start point to its end point. In this way, $\mathcal{G}$ can be viewed as a directed bush-type graph.

For such a directed bush-type graph $\mathcal{G}$, we refer to the set of edges with start (end) point $v$ as $E^{-}(v)\left(E^{+}(v)\right)$. The number of the elements in $E^{-}(v)\left(E^{+}(v)\right)$ is called the out-degree (in-degree) of $v$, denoted by $d^{-}(v)\left(d^{+}(v)\right)$. A vertex $v \in V$ is called a boundary vertex of $\mathcal{G}$ if $d^{-}(v)=0$ and an interior vertex otherwise. An edge is called a boundary edge if its end point is a boundary vertex. In particular, let $N_{B}$ be the number of boundary vertices, $V_{B}$ be the set of boundary vertices, $V_{I}$ be the set of interior vertices, and $V_{I T}=V_{I} \backslash V_{c}$.

Take Figure 1 as an example. In this concrete bush-type graph, $I_{T}=\{1,3\}, \mathcal{T}=\mathcal{T}_{1} \cup \mathcal{T}_{3} . N_{c}=6, N_{1}=N_{1,1}=$ $2, N_{3}=N_{3,1}+N_{3,2}=3+5=8$. The number of the edges (vertices) of the graph is $N=N_{c}+N_{1}+N_{3}=16$. The heights of the trees are $p_{1}=1, p_{3}=2$. The out-degrees of vertices $v_{1,1}^{3}$ and $v_{1,2}^{3}$ are $d^{-}\left(v_{1,1}^{3}\right)=2, d^{-}\left(v_{1,2}^{3}\right)=3$, and the out-degrees of the other vertices are 0 . Moreover, $V_{I T}=\left\{v_{1,1}^{3}, v_{1,2}^{3}\right\}$. The boundary vertex set is $V_{B}=\left\{v_{1,1}^{1}, v_{1,2}^{1}, v_{1,3}^{3}, v_{2, j}^{3}, j=1,2, \ldots, 5\right\}$. The number of the boundary vertices is $N_{B}=8$.

Now we assume that $\mathcal{G}$ is a geometric graph. For each $e \in E$, it has a finite arc length $\ell_{e}$. If $e \in E$ and $e=[v, \widehat{v}]$, then we parameterize it by its arc length via the function $\pi_{e}$ defined by

$$
\pi_{e}:\left[0, \ell_{e}\right] \rightarrow e
$$

where $\pi_{e}(0)=v, \pi_{e}\left(\ell_{e}\right)=\widehat{v}$.

Let $y(z)$ be a function defined on $E$. We defined the parameterization of $y(z)$ on each $e \in E$ by

$$
y_{e}(x)=y\left(\pi_{e}(x)\right), \quad x \in\left(0, \ell_{e}\right)
$$

where $x$ is the arc-length parameter.

Definition 2.1. Let $\mathcal{G}$ be a directed bush-type graph, and $y: \mathcal{G} \times \mathbb{R}_{+} \rightarrow \mathbb{C}$ be a scalar function in $(z, t) \in \mathcal{G} \times \mathbb{R}_{+}$. Suppose that the parameterization $y_{e}(x, t)$ of $y(z, t)$ on each edge $e \in \mathcal{G}$ satisfies the following wave equations

$$
m_{e} y_{e, t t}(x, t)=T_{e} y_{e, x x}(x, t), \quad x \in\left(0, \ell_{e}\right)
$$

Then we say that $y(z, t)$ satisfies 1 -D wave equation on $\mathcal{G}$, and $\mathcal{G}$ together with $y(z, t)$ is called a bush-type wave network, which is still denoted by $\mathcal{G}$ when there is no scope for ambiguity.

For the bush-type wave networks, in addition, we impose the following conditions at its interior vertices and boundary vertices:

(1) At every interior vertex, $\mathcal{G}$ satisfies the geometric continuity condition:

$$
y_{\widehat{e}}\left(\ell_{\widehat{e}}, t\right)=y_{\bar{e}}(0, t)=y(v, t), \quad v \in V_{I}, \widehat{e} \in E^{+}(v), \bar{e} \in E^{-}(v)
$$

where for each $e \in E, \lim _{x \rightarrow \ell_{e}^{-}} y_{e}(x, t)=y_{e}\left(\ell_{e}, t\right)$ and $\lim _{x \rightarrow 0^{+}} y_{e}(x, t)=y_{e}(0, t)$.

(2) The dynamic condition (the Kirchhoff law) at every interior vertex:

$$
T_{\widehat{e}} y_{\widehat{e}, x}\left(\ell_{\widehat{e}}, t\right)-\sum_{\bar{e} \in E^{-}(v)} T_{\bar{e}} y_{\bar{e}, x}(0, t)=-\alpha_{v} y_{t}(v, t)-\beta_{v} y(v, t), \quad v \in V_{I}, \widehat{e} \in E^{+}(v)
$$

(3) At every boundary vertex, we impose the dynamic condition:

$$
T_{\widehat{e}} y_{\widehat{e}, x}\left(\ell_{\widehat{e}}, t\right)=-\alpha_{v} y_{t}(v, t)-\beta_{v} y(v, t), \quad v \in V_{B}, \widehat{e} \in E^{+}(v) .
$$

In the sequel, we always assume that $\alpha_{v} \geq 0, \beta_{v} \geq 0$ for $v \in V_{I} \cup V_{B}$ and $\sum_{v \in V_{B}} \beta_{v}>0$. That is, the feedback law (2) is an internal feedback, and (3) is a boundary feedback with position term. 
Although the lengths of the edges may be distinct from one another, they can be normalized to 1 by a variable transform $x=\ell_{e} \tilde{x}$ for each edge $e \in E$. In fact, this variable transform leads (2.1) to

$$
\widetilde{m}_{e} \widetilde{y}_{e, t t}(\widetilde{x}, t)=\widetilde{T}_{e} \widetilde{y}_{e, \widetilde{x} \widetilde{x}}(\widetilde{x}, t), \quad \widetilde{x} \in(0,1)
$$

where $\widetilde{m}_{e}=m_{e} \ell_{e}, \widetilde{T}_{e}=T_{e} / \ell_{e}, \widetilde{y}_{e}(\widetilde{x}, t)=y_{e}(x, t)$. This shows that by normalizing $y$, the length $\ell_{e}$ of edge $e$ appears in the parameters $m_{e}$ and $T_{e}$. So without loss of generality we can assume that $y_{k}^{c}(x, t)$ and $y_{j, i}^{k}(x, t)$, $x \in(0,1)$ are the normalizations of $y(z, t)$ on edge $e_{k}^{c}$ and $e_{j, i}^{k}$, respectively. Thus a bush-type wave network $\mathcal{G}$ is described as

$$
\begin{gathered}
\left\{\begin{array}{l}
T_{k}^{c} y_{k, x x}^{c}(x, t)=m_{k}^{c} y_{k, t t}^{c}(x, t), \quad x \in(0,1) \\
T_{j, i}^{k} y_{j, i, x x}^{k}(x, t)=m_{j, i}^{k} y_{j, i, t t}^{k}(x, t), \quad x \in(0,1) \\
k \in I_{c}, j=1,2, \ldots, p_{k}, i=1,2, \ldots, N_{k, j}
\end{array}\right. \\
\left\{\begin{array}{l}
y_{k-1}^{c}(1, t)=y_{k}^{c}(0, t)=y_{1, i}^{k}(0, t)=y\left(v_{k}^{c}, t\right), \quad k \in I_{c}, i=1,2, \ldots, N_{k, 1} \\
y_{j, i}^{k}(1, t)=y_{j+1, l}^{k}(0, t)=y\left(v_{j, i}^{k}, t\right), \quad v_{j, i}^{k} \in V_{I T}, e_{j+1, l}^{k} \in E^{-}\left(v_{j, i}^{k}\right) \\
y_{j, i}^{k}(1, t)=y\left(v_{j, i}^{k}, t\right), \quad v_{j, i}^{k} \in V_{B}
\end{array}\right.
\end{gathered}
$$

and

$$
\left\{\begin{array}{l}
T_{k-1}^{c} y_{k-1, x}^{c}(1, t)-T_{k}^{c} y_{k, x}^{c}(0, t)-\sum_{i=1}^{N_{k, 1}} T_{1, i}^{k} y_{1, i, x}^{k}(0, t)=-\alpha_{k}^{c} y_{t}\left(v_{k}^{c}, t\right)-\beta_{k}^{c} y\left(v_{k}^{c}, t\right), \quad k \in I_{c} \\
T_{j, i}^{k} y_{j, i, x}^{k}(1, t)-\sum_{e_{j+1, l}^{k} \in E^{-}\left(v_{j, i}^{k}\right)} T_{j+1, l}^{k} y_{j+1, l, x}^{k}(0, t)=-\alpha_{j, i}^{k} y_{t}\left(v_{j, i}^{k}, t\right)-\beta_{j, i}^{k} y\left(v_{j, i}^{k}, t\right), \quad v_{j, i}^{k} \in V_{I T} \\
T_{j, i}^{k} y_{j, i, x}^{k}(1, t)=-\alpha_{j, i}^{k} y_{t}\left(v_{j, i}^{k}, t\right)-\beta_{j, i}^{k} y\left(v_{j, i}^{k}, t\right), \quad v_{j, i}^{k} \in V_{B} .
\end{array}\right.
$$

The system (2.2)-(2.4) is a general form of a bush-type wave network under consideration.

\subsection{Well-posedness of the system}

In this subsection, we give the well-posedness of the system (2.2)-(2.4) for the applications later. We first formulate (2.2)-(2.4) into an abstract evolutionary equation in an appropriate Hilbert space.

Let $y(z, t)$ be the function defined on $\mathcal{G} \times \mathbb{R}_{+}$. We associate it with a vector-valued function $Y(x, t)$, whose components are $y_{k}^{c}(x, t)$ and $y_{j, i}^{k}(x, t)$, ordered as below:

$$
\begin{gathered}
Y_{c}(x, t)=\left(y_{1}^{c}(x, t), y_{2}^{c}(x, t), \ldots, y_{N_{c}}^{c}(x, t)\right) \\
Y_{k}(x, t)=\left(y_{1,1}^{k}(x, t), \ldots, y_{1, N_{k, 1}}^{k}(x, t), y_{2,1}^{k}(x, t), \ldots, y_{2, N_{k, 2}}^{k}(x, t), \ldots, y_{p_{k}, 1}^{k}(x, t), \ldots, y_{p_{k}, N_{k, p_{k}}}^{k}(x, t)\right) \\
Y(x, t)=\left(Y_{c}(x, t), Y_{1}(x, t), Y_{2}(x, t), \ldots, Y_{N_{c}}(x, t)\right)^{T}
\end{gathered}
$$

where the superscript $T$ stands for the transpose of a matrix. We associate $y(v, t), v \in V$ with a vector-valued function $Y(V, t)$, whose components are $y\left(v_{k}^{c}, t\right)$ and $y\left(v_{j, i}^{k}, t\right)$, ordered in accordance with $Y(x, t)$.

Suppose that $y(z, t)$ satisfies the 1 -D wave equation on $\mathcal{G}$. We associate the coefficient functions with the matrices denoted by $T, M, \Gamma$ and $S$, respectively. They are diagonal matrices whose diagonal elements are $T_{k}^{c}, T_{j, i}^{k} ; m_{k}^{c}, m_{j, i}^{k} ; \alpha_{k}^{c}, \alpha_{j, i}^{k}$ and $\beta_{k}^{c}, \beta_{j, i}^{k}$, ordered in consistent with $Y(x, t)$, respectively.

Denote by $\Phi^{+}$and $\Phi^{-}$the incoming and outgoing incidence matrices of the directed bush-type graph $\mathcal{G}$, and $\Phi:=\Phi^{+}-\Phi^{-}$its incidence matrix (definitions of them are referred to any standard graph theory textbook, e.g. $[5,12]$ or the Appendix in our paper). 
With the help of the above notations we can rewrite the system (2.2)-(2.4) into the vector-valued form:

$$
\left\{\begin{array}{l}
T Y_{x x}(x, t)=M Y_{t t}(x, t), \quad x \in(0,1) \\
Y(1, t)=\left(\Phi^{+}\right)^{T} Y(V, t) \\
Y(0, t)=\left(\Phi^{-}\right)^{T} Y(V, t) \\
\Phi^{+} T Y_{x}(1, t)-\Phi^{-} T Y_{x}(0, t)=-\Gamma Y_{t}(V, t)-S Y(V, t)
\end{array}\right.
$$

Set

$$
\begin{gathered}
L^{2}(E)=\left\{f \mid f_{k}^{c} \in L^{2}(0,1), f_{j, i}^{k} \in L^{2}(0,1), k \in I_{c}, j=1,2, \ldots, p_{k}, i=1,2, \ldots, N_{k, j}\right\} \text { and } \\
H^{n}(E)=\left\{f \mid f_{k}^{c} \in H^{n}(0,1), f_{j, i}^{k} \in H^{n}(0,1), k \in I_{c}, j=1,2, \ldots, p_{k}, i=1,2, \ldots, N_{k, j}\right\}
\end{gathered}
$$

where $H^{n}(0,1)$ is the usual Sobolev space of order $n$, and

$$
H_{e}^{n}(\mathcal{G})=\left\{f \in H^{n}(E) \mid \exists \eta \in \mathbb{R}^{N} \text { such that } f(1)=\left(\Phi^{+}\right)^{T} \eta, f(0)=\left(\Phi^{-}\right)^{T} \eta\right\}
$$

Actually, $H_{e}^{n}(\mathcal{G})$ denotes the functions in $H^{n}(E)$ that satisfy the geometric continuity condition (1) at the interior vertices. For convenience, we denote by $f(V)=\eta$ if $\eta$ satisfies $f(1)=\left(\Phi^{+}\right)^{T} \eta$ and $f(0)=\left(\Phi^{-}\right)^{T} \eta$.

Let $\mathcal{H}$ be the state space defined by

$$
\mathcal{H}=H_{e}^{1}(\mathcal{G}) \times L^{2}(E)
$$

equipped with the inner product

$$
\langle(f, g),(\widehat{f}, \widehat{g})\rangle_{\mathcal{H}}=\int_{0}^{1}\left(T f^{\prime}(x), \widehat{f}^{\prime}(x)\right)_{\mathbb{C}^{N}} \mathrm{~d} x+\int_{0}^{1}(M g(x), \widehat{g}(x))_{\mathbb{C}^{N}} \mathrm{~d} x+(S f(V), \widehat{f}(V))_{\mathbb{C}^{N}}
$$

where $(\cdot, \cdot)_{\mathbb{C}^{N}}$ is the usual inner product in $\mathbb{C}^{N}, S$ is a nonnegative matrix, and the term $(S f(V), \widehat{f}(V))_{\mathbb{C}^{N}}$ is added to ensure that $\|(f, g)\|_{\mathcal{H}}:=\sqrt{\langle(f, g),(f, g)\rangle_{\mathcal{H}}}$ is a norm of $\mathcal{H}$. Clearly, $\mathcal{H}$ is a Hilbert space.

Define an operator $\mathcal{A}$ in $\mathcal{H}$ by

$$
\mathcal{A}(f, g)=\left(g, M^{-1} T f^{\prime \prime}\right), \quad(f, g) \in \mathcal{D}(\mathcal{A})
$$

with domain

$$
\mathcal{D}(\mathcal{A})=\left\{(f, g) \in \mathcal{H} \mid \begin{array}{c}
f \in H^{2}(E), g \in H_{e}^{1}(\mathcal{G}) \\
\Phi^{+} T f^{\prime}(1)-\Phi^{-} T f^{\prime}(0)=-\Gamma g(V)-S f(V)
\end{array}\right\} .
$$

Thus the equations (2.5) can be rewritten into an abstract evolutionary equation in $\mathcal{H}$ :

$$
\left\{\begin{array}{l}
\frac{\mathrm{d}}{\mathrm{d} t} W(t)=\mathcal{A} W(t), \quad t>0 \\
W(t)=\left(Y(x, t), Y_{t}(x, t)\right) \\
W(0)=\left(Y_{0}(x), Y_{1}(x)\right)
\end{array}\right.
$$

where $\left(Y_{0}(x), Y_{1}(x)\right) \in \mathcal{H}$ is an appropriate initial condition.

The following theorem gives the well-posedness of (2.9).

Theorem 2.1. Let $\mathcal{H}$ and $\mathcal{A}$ be defined by (2.6)-(2.8). Then $\mathcal{A}$ is a dissipative operator in $\mathcal{H}, 0 \in \rho(\mathcal{A})$, and $\mathcal{A}^{-1}$ is compact on $\mathcal{H}$. Hence $\mathcal{A}$ generates a $C_{0}$ semigroup $S(t)$ of contraction on $\mathcal{H}$.

Proof. A direct verification shows that $\mathcal{A}$ is a closed and densely defined linear operator in $\mathcal{H}$. The detail is omitted. In what follows, we mainly prove the other assertions. 
Firstly, $\mathcal{A}$ is a dissipative operator in $\mathcal{H}$. In fact, for any $(f, g) \in \mathcal{D}(\mathcal{A})$, we have

$$
\begin{aligned}
2 \Re\langle\mathcal{A}(f, g),(f, g)\rangle_{\mathcal{H}}= & \langle\mathcal{A}(f, g),(f, g)\rangle_{\mathcal{H}}+\langle(f, g), \mathcal{A}(f, g)\rangle_{\mathcal{H}} \\
= & \int_{0}^{1}\left(T g^{\prime}(x), f^{\prime}(x)\right)_{\mathbb{C}^{N}} \mathrm{~d} x+\int_{0}^{1}\left(T f^{\prime \prime}(x), g(x)\right)_{\mathbb{C}^{N}} \mathrm{~d} x+(S g(V), f(V))_{\mathbb{C}^{N}} \\
& +\int_{0}^{1}\left(f^{\prime}(x), T g^{\prime}(x)\right)_{\mathbb{C}^{N}} \mathrm{~d} x+\int_{0}^{1}\left(g(x), T f^{\prime \prime}(x)\right)_{\mathbb{C}^{N}} \mathrm{~d} x+(f(V), S g(V))_{\mathbb{C}^{N}} \\
= & \left(T g(1), f^{\prime}(1)\right)_{\mathbb{C}^{N}}-\left(T g(0), f^{\prime}(0)\right)_{\mathbb{C}^{N}}+\left(T f^{\prime}(1), g(1)\right)_{\mathbb{C}^{N}}-\left(T f^{\prime}(0), g(0)\right)_{\mathbb{C}^{N}} \\
& +(S g(V), f(V))_{\mathbb{C}^{N}}+(f(V), S g(V))_{\mathbb{C}^{N}} \\
= & \left(g(V), \Phi^{+} T f^{\prime}(1)-\Phi^{-} T f^{\prime}(0)+S f(V)\right)_{\mathbb{C}^{N}} \\
& +\left(\Phi^{+} T f^{\prime}(1)-\Phi^{-} T f^{\prime}(0)+S f(V), g(V)\right)_{\mathbb{C}^{N}} \\
= & -2(\Gamma g(V), g(V))_{\mathbb{C}^{N}}
\end{aligned}
$$

Since $\Gamma$ is a nonnegative matrix, so $\Re\langle\mathcal{A}(f, g),(f, g)\rangle_{\mathcal{H}}=-(\Gamma g(V), g(V))_{\mathbb{C}^{N}} \leq 0$, i.e., $\mathcal{A}$ is dissipative.

Next we prove that $\mathcal{A}^{-1}$ exists on $\mathcal{H}$. In fact, $\mathcal{A}$ is injective. This is because if there is a pair $(f, g) \in \mathcal{D}(\mathcal{A})$ such that $\mathcal{A}(f, g)=0$, then it holds that $g=0$ and $f$ satisfies the equations

$$
\left\{\begin{array}{l}
M^{-1} T f^{\prime \prime}(x)=0, \quad x \in(0,1) \\
f(1)=\left(\Phi^{+}\right)^{T} f(V), \quad f(0)=\left(\Phi^{-}\right)^{T} f(V) \\
\Phi^{+} T f^{\prime}(1)-\Phi^{-} T f^{\prime}(0)=-S f(V)
\end{array}\right.
$$

The differential equation implies that $f^{\prime}(x) \equiv c, x \in[0,1]$ where $c$ is a constant vector in $\mathbb{C}^{N}$. Integrating it over $[0,1]$, we get $f(1)=f(0)+c$. Substituting it into the boundary conditions yields $\left(S+\Phi T \Phi^{T}\right) f(V)=0$. Since $S+\Phi T \Phi^{T}$ is a positive definite matrix (see Appendix), we have $f(V)=0$, which leads to $f(x) \equiv 0, x \in[0,1]$. So $\mathcal{A}$ is injective.

Further, $\mathcal{A}$ is surjective. For any $(u, w) \in \mathcal{H}$, we consider the solvability of the resolvent equation $\mathcal{A}(f, g)=$ $(u, w)$, i.e., $g=u$ and

$$
T f^{\prime \prime}(x)=M w(x), \quad x \in(0,1) .
$$

For any test function $\varphi(x)$, taking inner product of $(2.10)$ and $\varphi(x)$ in $\mathbb{C}^{N}$, integrating it over $[0,1]$ and using integration by parts, we get

$$
\int_{0}^{1}(M w(x), \varphi(x))_{\mathbb{C}^{N}} \mathrm{~d} x=\left.\left(T f^{\prime}(x), \varphi(x)\right)_{\mathbb{C}^{N}}\right|_{0} ^{1}-\int_{0}^{1}\left(T f^{\prime}(x), \varphi^{\prime}(x)\right)_{\mathbb{C}^{N}} \mathrm{~d} x
$$

where

$$
\begin{aligned}
\left.\left(T f^{\prime}(x), \varphi(x)\right)_{\mathbb{C}^{N}}\right|_{0} ^{1} & =\left(T f^{\prime}(1), \varphi(1)\right)_{\mathbb{C}^{N}}-\left(T f^{\prime}(0), \varphi(0)\right)_{\mathbb{C}^{N}} \\
& =\left(\Phi^{+} T f^{\prime}(1), \varphi(V)\right)_{\mathbb{C}^{N}}-\left(\Phi^{-} T f^{\prime}(0), \varphi(V)\right)_{\mathbb{C}^{N}} \\
& =-(\Gamma u(V), \varphi(V))_{\mathbb{C}^{N}}-(S f(V), \varphi(V))_{\mathbb{C}^{N}}
\end{aligned}
$$

by assuming that $\varphi$ satisfies $\varphi(1)=\left(\Phi^{+}\right)^{T} \varphi(V), \varphi(0)=\left(\Phi^{-}\right)^{T} \varphi(V)$.

Now we define a bilinear functional $B\left[\psi_{1}, \psi_{2}\right]$ on $H_{e}^{1}(\mathcal{G})$ by

$$
B\left[\psi_{1}, \psi_{2}\right]=\int_{0}^{1}\left(T \psi_{1}^{\prime}(x), \psi_{2}^{\prime}(x)\right)_{\mathbb{C}^{N}} \mathrm{~d} x+\left(S \psi_{1}(V), \psi_{2}(V)\right)_{\mathbb{C}^{N}}, \quad \forall \psi_{1}, \psi_{2} \in H_{e}^{1}(\mathcal{G}) .
$$


Obviously,

$$
B[\psi, \psi]=\int_{0}^{1}\left(T \psi^{\prime}(x), \psi^{\prime}(x)\right)_{\mathbb{C}^{N}} \mathrm{~d} x+(S \psi(V), \psi(V))_{\mathbb{C}^{N}}:=\|\psi\|_{H_{e}^{1}(\mathcal{G})}
$$

is an equivalent norm on $H_{e}^{1}(\mathcal{G})$, and $\left|B\left[\psi_{1}, \psi_{2}\right]\right| \leq\left\|\psi_{1}\right\|_{H_{e}^{1}(\mathcal{G})}|| \psi_{2} \|_{H_{e}^{1}(\mathcal{G})}$. So $B$ is a coercive and bounded bilinear functional on $H_{e}^{1}(\mathcal{G})$. Then by Lax-Milgram's lemma, there is unique a solution $f \in H_{e}^{1}(\mathcal{G})$ satisfying $(2.11)$, and hence $f$ satisfies $(2.10)$, which implies $f \in H^{2}(E)$. Since we have used

$$
\Phi^{+} T f^{\prime}(1)-\Phi^{-} T f^{\prime}(0)=-\Gamma g(V)-S f(V)=-\Gamma u(V)-S f(V)
$$

in $(2.12)$, so it holds that $(f, g)=(f, u) \in \mathcal{D}(\mathcal{A})$. So $\mathcal{A}$ is surjective.

The inverse operator theorem asserts that $\mathcal{A}^{-1}$ is bounded, i.e., $0 \in \rho(\mathcal{A})$. Note that $\mathcal{D}(\mathcal{A}) \subset H^{2}(E) \times H_{e}^{1}(\mathcal{G})$, so the Sobolev Embedding Theorem ensures that $\mathcal{A}^{-1}$ is a compact operator on $\mathcal{H}$. Finally the assertion that $\mathcal{A}$ generates a $C_{0}$ semigroup $S(t)$ of contraction on $\mathcal{H}$ follows from Lumer-Phillips Theorem [18].

\section{Controller design of Bush-type network - the "Cutting-edge" method}

In this section, we study the controller design and stability of the closed loop system. The controller design means that we choose the coefficients $\alpha_{v}$ and $\beta_{v}$ in (2.2)-(2.4), of which some precisely take positive values, and the others are 0 . The choice approach is based on the so-called "cutting-edge" method. The design procedure is the same as the one described in Section 1. The purpose of the controller design is to stabilize the system. The main result is stated in Theorem 3.1 in Section 3.1; its precise proof in theory is given in Section 3.2 by the spectral analysis approach.

\subsection{Main result}

In this subsection we state the control strategy for the system (2.2)-(2.4). To simplify the statement, we need the following definition.

Definition 3.1. (i) If there exist two successive edges $e_{k}^{c}, e_{k+1}^{c}$ in the cycle $\mathcal{C}$ such that the wave speed ratio $\frac{\sqrt{m_{k}^{c} / T_{k}^{c}}}{\sqrt{m_{k+1}^{c} / T_{k+1}^{c}}}$ is irrational, then we say that the cycle $\mathcal{C}$ satisfies condition $(\mathrm{A})$, or precisely, the edge pair $\left(e_{k}^{c}, e_{k+1}^{c}\right)$ satisfies condition $(\mathrm{A})$;

(ii) By imposing the boundary control on $\mathcal{G}$, we mean that all the boundary vertices are equipped with velocity feedback controllers, i.e., $\alpha_{j, i}^{k}>0, \forall v_{j, i}^{k} \in V_{B}$;

(iii) By imposing an interior nodal controller at $v_{k}^{c}, k \in I_{c} \backslash I_{T}$, we mean that a velocity feedback controller is equipped at the interior vertex $v_{k}^{c}$ on cycle $\mathcal{C}$, which is not a root of some tree, i.e., $\alpha_{k}^{c}>0, k \in I_{c} \backslash I_{T}$;

(iv) By imposing an interior point controller at $q$ on $e_{k}^{c}, k \in I_{c}$, we mean that a velocity feedback controller is equipped at the point $q$ on the interior edge $e_{k}^{c}$ of cycle $\mathcal{C}$, i.e., the equations on edge $e_{k}^{c}$ change into

$$
\left\{\begin{array}{l}
T_{k}^{c} y_{k, x x}^{c}(x, t)=m_{k}^{c} y_{k, t t}^{c}(x, t), \quad x \in(0, q) \cup(q, 1) \\
y_{k}^{c}\left(q^{-}, t\right)=y_{k}^{c}\left(q^{+}, t\right) \\
T_{k}^{c} y_{k, x}^{c}\left(q^{-}, t\right)-T_{k}^{c} y_{k, x}^{c}\left(q^{+}, t\right)=-\alpha_{k, q}^{c} y_{k, t}^{c}(q, t)
\end{array}\right.
$$

where $\alpha_{k, q}^{c}>0$ and $q \in(0,1)$ such that $q /(1-q)$ is irrational.

The interior nodal controller and the interior point controller are said to be interior controllers.

The theorem below gives the main result of this paper, which includes the strategy of the controller design, i.e., the number and locations of the feedback controllers for a bush-type wave network.

Theorem 3.1 (main theorem). Let $\mathcal{G}=\mathcal{C} \cup \mathcal{T}$ be a bush-type graph, and the wave system on $\mathcal{G}$ be given by (2.2)-(2.4). Firstly, we impose the boundary control on $\mathcal{G}$, which has at most $N_{B}$ controllers equipped 
at the boundary vertices and only one $\beta_{v}>0$. Next, we impose at most three interior controllers at appropriate positions on cycle $\mathcal{C}$ in the following manner:

Case 1. If cycle $\mathcal{C}$ satisfies condition $(\mathrm{A})$, i.e., there is an edge pair $\left(e_{k}^{c}, e_{k+1}^{c}\right)$ satisfying condition $(\mathrm{A})$, then we impose at most three interior nodal controllers at their start and end points $v_{j}^{c}, j \in\{k, k+1, k+2\} \backslash I_{T}$, which are not the roots of the trees;

Case 2. If cycle $\mathcal{C}$ fails to satisfy condition $(\mathrm{A})$, then for one edge $e_{k}^{c}=\left[v_{k}^{c}, v_{k+1}^{c}\right]$ on $\mathcal{C}$, we impose an interior point controller at $q$ on $e_{k}^{c}$, and at most two interior nodal controllers at $v_{j}^{c}, j \in\{k, k+1\} \backslash I_{T}$.

With this control strategy, the system (2.2)-(2.4) is asymptotically stabilized by at most $N_{B}+3$ controllers.

Remark 3.1. In the control strategy, we choose only one $\beta_{v}>0$ at a boundary vertex, which aims to remove the zero eigenvalue. According to the control strategy given in Theorem 3.1, $N_{B}+3$ controllers is a sufficient condition to stabilize asymptotically the bush-type wave networks, but not a necessary one. In fact, for a concrete bush-type graph, we can use fewer than $N_{B}+3$ controllers to stabilize the system. The examples will be given in Section 4 .

\subsection{Proof of the main result}

In this subsection, we give a complete proof of Theorem 3.1, especially, the asymptotic stability of the closed loop system. Our proof is based on Theorem 1.1. We shall show that each tree corresponds to an invariant subspace similar to $X_{1}$ described in Theorem 1.1, of which the proof includes the idea of "cutting-edge". By the decomposition of space $\mathcal{H}$, we can "cut" all the trees in the graph under the boundary control. Finally we prove that the three interior controllers can stabilize the rest cycle. The whole proof is complete by the spectral analysis approach. Since the proof is very long, we finish it by three steps. The first step decomposes the graph; in the second step we "cut" each tree; and in the third step we "cut" the rest cycle.

\section{Step 1. Decomposition of $\mathcal{G}$}

Here we shall give a division of the bush-type graph $\mathcal{G}$, which will correspond with the decomposition of space $\mathcal{H}$ under the control strategy.

According to the geometric structure of a bush-type graph, we separate it into the following parts:

$$
\mathcal{G}=\cup_{k \in I_{T}} \mathcal{T}_{k} \cup \mathcal{C} .
$$

Introduce the following spaces defined on tree $\mathcal{T}_{k}, k \in I_{T}$ and cycle $\mathcal{C}$ :

$$
\begin{gathered}
L^{2}\left(E_{k}\right)=\left\{f=\left\{f_{j, i}^{k}\right\} \mid f_{j, i}^{k} \in L^{2}(0,1), e_{j, i}^{k} \in E_{k}\right\}, \quad L^{2}\left(E_{c}\right)=\left\{f=\left\{f_{k}^{c}\right\} \mid f_{k}^{c} \in L^{2}(0,1), k \in I_{c}\right\} \\
H^{n}\left(E_{k}\right)=\left\{f=\left\{f_{j, i}^{k}\right\} \mid f_{j, i}^{k} \in H^{n}(0,1), e_{j, i}^{k} \in E_{k}\right\}, \quad H^{n}\left(E_{c}\right)=\left\{f=\left\{f_{k}^{c}\right\} \mid f_{k}^{c} \in H^{n}(0,1), k \in I_{c}\right\} \\
H_{e}^{n}\left(\mathcal{T}_{k}\right)=\left\{f \in H^{n}\left(E_{k}\right) \mid f(1)=\left(\Phi_{k}^{+}\right)^{T} f\left(V_{k}\right), f(0)=\left(\Phi_{k}^{-}\right)^{T} f\left(V_{k}\right)\right\} \\
H_{e}^{n}(\mathcal{C})=\left\{f \in H^{n}\left(E_{c}\right) \mid f(1)=\left(\Phi_{c}^{+}\right)^{T} f\left(V_{c}\right), f(0)=\left(\Phi_{c}^{-}\right)^{T} f\left(V_{c}\right)\right\}
\end{gathered}
$$

where $\Phi_{k}^{+}$and $\Phi_{k}^{-}$are the incoming and outgoing matrices of tree $\mathcal{T}_{k} ; \Phi_{c}^{+}$and $\Phi_{c}^{-}$are the incoming and outgoing matrices of cycle $\mathcal{C}$.

Similar as $H_{e}^{n}(\mathcal{G})$, the space $H_{e}^{n}\left(\mathcal{T}_{k}\right)$ denotes the functions in $H^{n}\left(E_{k}\right)$ that satisfy the geometric continuity condition at vertices in $V_{k} \cap V_{I T}$; and $H_{e}^{n}(\mathcal{C})$ denotes the functions in $H^{n}\left(E_{c}\right)$ that satisfy the geometric continuity condition at vertices in $V_{c}$.

Let the state spaces $\mathcal{H}_{k}$ and $\mathcal{H}_{c}$ corresponding to the tree $\mathcal{T}_{k}, k \in I_{T}$ and the cycle $\mathcal{C}$ be as follows:

$$
\mathcal{H}_{k}=\mathcal{H}_{e}^{1}\left(\mathcal{T}_{k}\right) \times L^{2}\left(E_{k}\right), \quad \mathcal{H}_{c}=\mathcal{H}_{e}^{1}(\mathcal{C}) \times L^{2}\left(E_{c}\right) .
$$

Then we have

$$
\mathcal{H} \subset \bigoplus_{k \in I_{T}} \mathcal{H}_{k} \bigoplus \mathcal{H}_{c}
$$


This decomposition is corresponding to the graph division.

Let $S(t)$ be the $C_{0}$ semigroup generated by $\mathcal{A}$. We shall show that the invariant stable space described in Theorem 1.1 with property

$$
H_{s}=\left\{f \in \mathcal{H} \mid \lim _{t \rightarrow \infty} S(t) f=\lim _{t \rightarrow \infty} S^{*}(t) f=0\right\}
$$

will be given by $\left\{f \in \mathcal{H} \mid f\left(v_{k}^{c}\right)=0, k \in I_{T}\right\}$ under the boundary control. The proof is based on a stability theorem according to Lyubich and Phóng's theorem [16].

Lemma 3.1. Let $X$ be a Banach space, and let $A$ generate a uniformly bounded $C_{0}$ semigroup $T(t)$ on $X$. If the spectrum of $A$ satisfies $\sigma(A) \subset\{\lambda \mid \Re \lambda<0\}$, then the semigroup $T(t)$ is asymptotically stable.

For our problem, the operator $\mathcal{A}$ is resolvent compact, hence $\sigma(\mathcal{A})$ consists of isolated eigenvalues with finite multiplicities. By Lemma 3.1, it suffices to verify that there is no eigenvalue of $\mathcal{A}$ on the imaginary axis $i \mathbb{R}$. Since Theorem 2.1 says that $0 \in \rho(\mathcal{A})$, so we only need to prove that for any $\lambda \in i \mathbb{R}, \lambda \neq 0$, if there exists $(f, g) \in \mathcal{D}(\mathcal{A})$ such that $\mathcal{A}(f, g)=\lambda(f, g)$, then it must be $(f, g)=0$.

Step 2. "Cutting-edge" of the trees $\mathcal{T}_{k}, k \in I_{T}$

In what follows, we assume that for $\lambda \in i \mathbb{R}, \lambda \neq 0$, there exists $(f, g) \in \mathcal{D}(\mathcal{A})$ such that $\mathcal{A}(f, g)=\lambda(f, g)$. For any fixed $k \in I_{T}$, denote by $\left(f_{k}, g_{k}\right)$ the restriction of $(f, g)$ on the tree $\mathcal{T}_{k}$. We shall prove that under the boundary control, we have $f_{k}(x)=g_{k}(x) \equiv 0$. The procedure of the proof is a process of "cutting-edge" from the boundary edges to the cycle.

Due to $\mathcal{A}(f, g)=\lambda(f, g)$, we have $g_{k}=\lambda f_{k}$ and

$$
\left\{\begin{array}{l}
f_{j, i}^{k}{ }^{\prime \prime}(x)=\left(\rho_{j, i}^{k}\right)^{2} \lambda^{2} f_{j, i}^{k}(x), \quad x \in(0,1) \\
f_{1, i}^{k}(0)=f\left(v_{k}^{c}\right), \quad i=1,2, \ldots, N_{k, 1} \\
f_{j, i}^{k}(1)=f_{j+1, l}^{k}(0)=f\left(v_{j, i}^{k}\right), \quad v_{j, i}^{k} \in V_{I T}, e_{j+1, l}^{k} \in E^{-}\left(v_{j, i}^{k}\right) \\
f_{j, i}^{k}(1)=f\left(v_{j, i}^{k}\right), \quad v_{j, i}^{k} \in V_{B} \\
T_{j, i}^{k} f_{j, i}^{k}{ }^{\prime}(1)-\sum_{e_{j+1, l}^{k} \in E^{-}\left(v_{j, i}^{k}\right)} T_{j+1, l}^{k} f_{j+1, l}^{k} \quad{ }^{\prime}(0)=0, \quad v_{j, i}^{k} \in V_{I T} \\
T_{j, i}^{k} f_{j, i}^{k}{ }^{\prime}(1)=-\alpha_{j, i}^{k} g\left(v_{j, i}^{k}\right)-\beta_{j, i}^{k} f\left(v_{j, i}^{k}\right), \quad v_{j, i}^{k} \in V_{B}
\end{array}\right.
$$

where $\rho_{j, i}^{k}=\sqrt{m_{j, i}^{k} / T_{j, i}^{k}}$.

Note that

$$
0=\Re \lambda\langle(f, g),(f, g)\rangle_{\mathcal{H}}=\Re\langle\mathcal{A}(f, g),(f, g)\rangle_{\mathcal{H}}=-(\Gamma g(V), g(V))_{\mathbb{C}^{N}} .
$$

According to the control strategy in Theorem 3.1, we have $\alpha_{j, i}^{k}>0$ for all the boundary vertices $v_{j, i}^{k} \in V_{B}$. Thus the above equality implies that $g\left(v_{j, i}^{k}\right)=g_{j, i}^{k}(1)=0, v_{j, i}^{k} \in V_{B}$, and hence $f_{j, i}^{k}(1)=f\left(v_{j, i}^{k}\right)=0, v_{j, i}^{k} \in V_{B}$. The last equation in $(3.1)$ reads that $f_{j, i}^{k}{ }^{\prime}(1)=0$. Therefore on the boundary edges $e_{j, i}^{k}, f_{j, i}^{k}$ satisfy the differential equation

$$
\left\{\begin{array}{l}
f_{j, i}^{k}{ }^{\prime \prime}(x)=\left(\rho_{j, i}^{k}\right)^{2} \lambda^{2} f_{j, i}^{k}(x), \quad x \in(0,1) \\
f_{j, i}^{k}(1)=f_{j, i}^{k}{ }^{\prime}(1)=0, \quad i=1,2, \ldots, N_{k, j} .
\end{array}\right.
$$

The uniqueness theory of the ordinary differential equation asserts that (3.2) has unique a zero solution.

Now we consider the $p_{k}$-order edges of the tree $\mathcal{T}_{k}$. They are boundary edges of $\mathcal{T}_{k}$ according to the definition of $p_{k}$. Thanks to the previous argument, we have $f_{p_{k}, i}^{k}(x) \equiv 0, i=1,2, \ldots, N_{k, p_{k}}$. So we can "cut" these edges from $\mathcal{T}_{k}$.

Next we consider the $\left(p_{k}-1\right)$-order edges of $\mathcal{T}_{k}$, whose end points are $v_{p_{k}-1, i}^{k}, i=1,2, \ldots, N_{k, p_{k}-1}$. If $v_{p_{k}-1, i}^{k} \in V_{B}$, then we already have $f_{p_{k}-1, i}^{k}(x) \equiv 0$. If $v_{p_{k}-1, i}^{k} \in V_{I}$, then it is the start point of some $p_{k^{-o r d e r}}$ edges. Since we have derived that $f_{p_{k}, l}^{k}(x) \equiv 0$, the connective conditions in $(3.1)$ indicate that $f_{p_{k}-1, i}^{k}(1)=$ 
$f_{p_{k}-1, i}^{k}(1)=0$. Again using the uniqueness theory of the ordinary differential equation we get $f_{p_{k}-1, i}^{k}(x) \equiv 0$. Thus we can "cut" all the $\left(p_{k}-1\right)$-order edges from $\mathcal{T}_{k}$.

Generally, suppose that we have obtained

$$
f_{j+1, i}^{k}(x)=\ldots=f_{p_{k}-1, i}^{k}(x)=f_{p_{k}, i}^{k}(x) \equiv 0,
$$

which means that we have "cut" all the edges of $\mathcal{T}_{k}$ whose orders are larger than $j$. Then for the $j$-order edges of $\mathcal{T}_{k}$, whose end points are $v_{j, i}^{k}$, if $v_{j, i}^{k} \in V_{B}$, then we already have $f_{j, i}^{k}(x) \equiv 0$. If $v_{j, i}^{k} \in V_{I}$, then it is the start point of some $(j+1)$-order edges. The connective conditions in (3.1) yield that $f_{j}^{k}(1)=f_{j}^{k^{\prime}}(1)=0$. For the same reason as before, we have $f_{j, i}^{k}(x) \equiv 0$. So we can also "cut" all the $j$-order edges of $\mathcal{T}_{k}$. $(0,0)$.

By induction, we get $f_{k}(x) \equiv 0$. By now we have "cut" all the edges of the tree $\mathcal{T}_{k}$. Therefore $\left(f_{k}(x), g_{k}(x)\right) \equiv$

\section{Step 3. "Cutting-edge" of the cycle $\mathcal{C}$}

When we "cut" all the edges of the trees in $\mathcal{G}$, we readily have $f\left(v_{k}^{c}\right)=0, k \in I_{T}$, where $(f, g)$ is given in Step 2. Now we show that under the control strategy stated in the two cases in Theorem 3.1, the rest cycle can also be "cut".

Denote by $\left(f^{c}, g^{c}\right)$ the restriction of $(f, g)$ on the cycle $\mathcal{C}$, then $g^{c}=\lambda f^{c}$, and

$$
\left\{\begin{array}{l}
f_{k}^{c^{\prime \prime}}(x)=\left(\rho_{k}^{c}\right)^{2} \lambda^{2} f_{k}^{c}(x), \quad x \in(0,1), k \in I_{c} \\
f_{k-1}^{c}(1)=f_{k}^{c}(0)=f\left(v_{k}^{c}\right), \quad k \in I_{c} \\
T_{k-1}^{c} f_{k-1}^{c}{ }^{\prime}(1)-T_{k}^{c} f_{k}^{c^{\prime}}(0)-\sum_{i=1}^{N_{k, 1}} T_{1, i}^{k} f_{1, i}^{k}{ }^{\prime}(0)=-\alpha_{k}^{c} g\left(v_{k}^{c}\right), \quad k \in I_{c}
\end{array}\right.
$$

where $\rho_{k}^{c}=\sqrt{m_{k}^{c} / T_{k}^{c}}$.

Since we have "cut" all the trees, then at the vertices $v_{k}^{c}, k \in I_{T}$, we have

$$
\begin{gathered}
f_{k-1}^{c}(1)=f_{k}^{c}(0)=f\left(v_{k}^{c}\right)=g\left(v_{k}^{c}\right)=0, \quad k \in I_{T}, \quad \text { and } \\
f_{1, i}^{k}{ }^{\prime}(0)=0, \quad i=1,2, \ldots, N_{k, 1}, \quad k \in I_{T} .
\end{gathered}
$$

Let $I_{\Gamma}=\left\{k \in I_{c} \backslash I_{T} \mid \alpha_{k}^{c}>0\right\}$. Thanks to the equality

$$
0=\Re \lambda\langle(f, g),(f, g)\rangle_{\mathcal{H}}=\Re\langle\mathcal{A}(f, g),(f, g)\rangle_{\mathcal{H}}=-(\Gamma g(V), g(V))_{\mathbb{C}^{N}},
$$

we also have

$$
f_{k-1}^{c}(1)=f_{k}^{c}(0)=f\left(v_{k}^{c}\right)=g\left(v_{k}^{c}\right)=0, \quad k \in I_{\Gamma} .
$$

The above lead to the equations

$$
\left\{\begin{array}{l}
f_{k}^{c \prime \prime}(x)=\left(\rho_{k}^{c}\right)^{2} \lambda^{2} f_{k}^{c}(x), \quad x \in(0,1), \quad k \in I_{c} \\
f_{k-1}^{c}(1)=f_{k}^{c}(0)=f\left(v_{k}^{c}\right)=0, \quad k \in I_{T} \cup I_{\Gamma} \\
f_{k-1}^{c}(1)=f_{k}^{c}(0)=f\left(v_{k}^{c}\right), \quad k \in I_{c} \backslash\left\{I_{T} \cup I_{\Gamma}\right\} \\
T_{k-1}^{c} f_{k-1}^{c}{ }^{\prime}(1)-T_{k}^{c} f_{k}^{c \prime}(0)=0, \quad k \in I_{c}
\end{array}\right.
$$

From (3.5) we see that if there is one $k_{0} \in I_{c}$ such that $f_{k_{0}}^{c}(x) \equiv 0$, then $f^{c}(x) \equiv 0$, and hence $\left(f^{c}, g^{c}\right)=(0,0)$. Therefore we need only to prove the existence of such a $k_{0}$ under the control strategy. In the following, we verify this in the two cases listed in Theorem 3.1. 


\section{Case 1. The cycle $\mathcal{C}$ satisfies condition (A)}

If the cycle $\mathcal{C}$ satisfies condition (A), according to the control strategy stated in Theorem 3.1, for some $k \in I_{c}$, we have $\{k, k+1, k+2\} \in I_{T} \cup I_{\Gamma}$, where $I_{\Gamma}=\left\{k \in I_{c} \backslash I_{T} \mid \alpha_{k}^{c}>0\right\}$. That is, each $v_{j}^{c}, j \in\{k, k+1, k+2\}$ is either equipped with an interior nodal controller or a root of some tree. In this case, we have

$$
\left\{\begin{array}{l}
f_{j}^{c \prime \prime}(x)=\left(\rho_{j}^{c}\right)^{2} \lambda^{2} f_{j}^{c}(x), \quad x \in(0,1), \quad j=k, k+1 \\
f_{k}^{c}(0)=f_{k+1}^{c}(0)=f_{k}^{c}(1)=f_{k+1}^{c}(1)=0 \\
T_{k}^{c} f_{k}^{c^{\prime}}(1)-T_{k+1}^{c} f_{k+1}^{c}(0)=0 .
\end{array}\right.
$$

Note that the general solution of the differential equation in (3.6) is of the form

$$
f_{j}^{c}(x)=a_{j}^{c} \cosh \lambda \rho_{j}^{c} x+b_{j}^{c} \sinh \lambda \rho_{j}^{c} x, \quad j=k, k+1
$$

where $a_{j}^{c}, b_{j}^{c} \in \mathbb{C}$ are constant coefficients.

Substituting (3.7) into the boundary conditions in (3.6) yields

$$
\left\{\begin{array}{l}
a_{k}^{c}=a_{k+1}^{c}=0 \\
b_{k}^{c} \sinh \lambda \rho_{k}^{c}=b_{k+1}^{c} \sinh \lambda \rho_{k+1}^{c}=0 \\
T_{k}^{c} \rho_{k}^{c} b_{k}^{c} \cosh \lambda \rho_{k}^{c}=T_{k+1}^{c} \rho_{k+1}^{c} b_{k+1}^{c} .
\end{array}\right.
$$

Since the cycle $\mathcal{C}$ satisfies condition (A), so $\sinh \lambda \rho_{k}^{c}$ and $\sinh \lambda \rho_{k+1}^{c}$ never vanish simultaneously, which implies that at least one of $b_{k}^{c}$ and $b_{k+1}^{c}$ is 0 . Hence at least one of $f_{k}^{c}(x)$ and $f_{k+1}^{c}(x)$ is a zero function. Therefore we have $f^{c}(x) \equiv 0$. This means that we can "cut" all the edges of the rest cycle. The desired result follows.

\section{Case 2. The cycle $\mathcal{C}$ fails to satisfy condition (A)}

If the cycle $\mathcal{C}$ fails to satisfy condition $(\mathrm{A})$, then for any two edges $e_{i}^{c}, e_{j}^{c}$ of $\mathcal{C}$, the wave speed ratio $\rho_{j}^{c} / \rho_{i}^{c}$ is rational. In this case, it is not sufficient to stabilize the system even if all the interior nodal controllers are equipped. According to the control strategy stated in Theorem 3.1, we choose an edge $e_{k}^{c}=\left[v_{k}^{c}, v_{k+1}^{c}\right]$ of $\mathcal{C}$, and an interior point $v_{q} \in\left(v_{k}^{c}, v_{k+1}^{c}\right)$ such that $q /(1-q)$ is irrational, where $q \in(0,1)$ is the arc length between $v_{k}^{c}$ and $v_{q}$. Then we impose at most three interior controllers at $v_{k}^{c}, v_{k+1}^{c}$ and $v_{q}$, which are the collocated velocity feedback controllers. Under this control strategy, we assert that $f_{k}^{c}(x) \equiv 0$.

Firstly we observe that under this control strategy, the system equation on the edge $e_{k}^{c}$ is given by

$$
\left\{\begin{array}{l}
m_{k}^{c} y_{k, t t}^{c}(x, t)=T_{k}^{c} y_{k, x x}^{c}(x, t), \quad x \in(0, q) \cup(q, 1) \\
y_{k}^{c}\left(q^{-}, t\right)=y_{k}^{c}\left(q^{+}, t\right)=y\left(v_{q}, t\right) \\
T_{k}^{c} y_{k, x}^{c}\left(q^{-}, t\right)-T_{k}^{c} y_{k, x}^{c}\left(q^{+}, t\right)=-\alpha_{q} y_{t}\left(v_{q}, t\right) \\
y_{k}^{c}(0, t)=y_{k-1}^{c}(1, t)=y\left(v_{k}^{c}, t\right) \\
T_{k-1}^{c} y_{k-1, x}^{c}(1, t)-T_{k}^{c} y_{k, x}^{c}(0, t)-\sum_{i=1}^{N_{k, 1}} T_{1, i}^{k} y_{1, i, x}^{k}(0, t)=-\alpha_{k}^{c} y_{t}\left(v_{k}^{c}, t\right) \\
y_{k}^{c}(1, t)=y_{k+1}^{c}(0, t)=y\left(v_{k+1}^{c}, t\right) \\
T_{k}^{c} y_{k, x}^{c}(1, t)-T_{k+1}^{c} y_{k+1, x}^{c}(0, t)-\sum_{i=1}^{N_{k+1,1}} T_{1, i}^{k+1} y_{1, i, x}^{k+1}(0, t)=-\alpha_{k+1}^{c} y_{t}\left(v_{k+1}^{c}, t\right) .
\end{array}\right.
$$


By the variable transforms $\widetilde{x}=\frac{x}{q}, x \in(0, q)$ and $\widehat{x}=\frac{x-q}{1-q}, x \in(q, 1)$, we can transform the system (3.8) into the standard form

$$
\left\{\begin{array}{l}
m_{k, q^{-}}^{c} y_{k, q^{-}, t t}^{c}(\widetilde{x}, t)=T_{k, q^{-}}^{c} y_{k, q^{-}, \widetilde{x} \widetilde{x}}^{c}(\widetilde{x}, t), \quad \widetilde{x} \in(0,1) \\
m_{k, q^{+}}^{c} y_{k, q^{+}, t t}^{c}(\widehat{x}, t)=T_{k, q^{+}}^{c} y_{k, q^{+}, \widehat{x} \widehat{x}}^{c}(\widehat{x}, t), \quad \widehat{x} \in(0,1) \\
y_{k, q^{-}}^{c}(1, t)=y_{k, q^{+}}^{c}(0, t)=y\left(v_{q}, t\right) \\
T_{k, q^{-}}^{c} y_{k, q^{-}, \widetilde{x}}^{c}(1, t)-T_{k, q^{+}}^{c} y_{k, q^{+}, \widehat{x}}^{c}(0, t)=-\alpha_{q} y\left(v_{q}, t\right) \\
y_{k, q^{-}}^{c}(0, t)=y_{k-1}^{c}(1, t)=y\left(v_{k}^{c}, t\right) \\
T_{k-1}^{c} y_{k-1, x}^{c}(1, t)-T_{k, q^{-}}^{c} y_{k, q^{-}, \widetilde{x}}^{c}(0, t)-\sum_{i=1}^{N_{k, 1}} T_{1, i}^{k} y_{1, i, x}^{k}(0, t)=-\alpha_{k}^{c} y_{t}\left(v_{k}^{c}, t\right) \\
y_{k, q^{+}}^{c}(1, t)=y_{k+1}^{c}(0, t)=y\left(v_{k+1}^{c}, t\right) \\
T_{k, q^{+}}^{c} y_{k, q^{+}, \widehat{x}}^{c}(1, t)-T_{k+1}^{c} y_{k+1, x}^{c}(0, t)-\sum_{i=1}^{N_{k+1,1}} T_{1, i}^{k+1} y_{1, i, x}^{k+1}(0, t)=-\alpha_{k+1}^{c} y_{t}\left(v_{k+1}^{c}, t\right)
\end{array}\right.
$$

where the coefficients are $T_{k, q^{-}}^{c}=T_{k}^{c} / q, m_{k, q^{-}}^{c}=m_{k}^{c} q, T_{k, q^{+}}^{c}=T_{k}^{c} /(1-q), m_{k, q^{+}}^{c}=m_{k}^{c}(1-q)$, and the functions are $y_{k, q^{-}}^{c}(\widetilde{x}, t)=y_{k}^{c}(x, t), x \in(0, q)$ and $y_{k, q^{+}}^{c}(\widehat{x}, t)=y_{k}^{c}(x, t), x \in(q, 1)$.

Denote by $\mathcal{C}_{q}$ the cycle $\mathcal{C}$ added a vertex $v_{q}$ at $q$ on edge $e_{k}^{c}$. The graph $\mathcal{G}_{q}=\mathcal{C}_{q} \cup \mathcal{T}$ is also of bush-type. The wave system on $\mathcal{G}_{q}$ with an interior point controller at $q$ falls into the systems described by (2.2)-(2.4), which has the same structure as the old one under the control strategy stated in Theorem 3.1. In particular, $\mathcal{C}_{q}$ satisfies condition (A). According to Case 1 , we have $f_{k}^{c}(x) \equiv 0$. So we can "cut" all the edges of $\mathcal{C}_{q}$.

To summarize the result of Step 1 to Step 3, we have proved that $i \mathbb{R} \cap \sigma(\mathcal{A})=\emptyset$ by "cutting-edge" method. So Lemma 3.1 indicates that the system (2.2)-(2.4) is asymptotically stable under the control strategy shown in Theorem 3.1. The proof is then complete.

Remark 3.2. From the proof above we see that the boundary controllers aim to stabilize the wave equation on the trees of $\mathcal{G}$, and the three interior controllers aim to stabilize the wave equation on the rest cycle. Such a control strategy can asymptotically stabilize the wave system on bush-type graphs. The proof is completed by "cutting-edge" step by step from the boundary edges of the trees $\mathcal{T}_{k}$ to the cycle $\mathcal{C}$. This method can also be used in the controller design for other complex graphs.

\section{EXAMPLES}

In this section, we give some examples of bush-type graph. The 1-D wave systems on these graphs fall into the model in Theorem 3.1. Here we address that for a concrete graph, we use fewer than $N_{B}+3$ controllers to stabilize the wave system asymptotically.

In figures shown in this section, the small rings stand for the controllers at vertices; the cross stands for the interior point controller at $q$ on some edge.

\subsection{Example 1}

In this example, the graph $\mathcal{G}$ is shown in Figure 2.

Step 1. Impose the boundary controllers.

Step 2. If there is an edge pair of the cycle satisfying condition (A), and one of the start and end points of the two edges is the root of some tree, then only two interior nodal controllers at vertices of the cycle are needed to stabilize the system asymptotically. For example, if the edge pair $\left(e_{8}^{c}, e_{1}^{c}\right)$ satisfies condition (A), then we only need to add two interior nodal controllers at the start points $v_{1}^{c}$ and $v_{8}^{c}$ of $e_{1}^{c}$ and $e_{8}^{c}$, since the end point $v_{2}^{c}$ of $e_{1}^{c}$ is a root, i.e., $v_{2}^{c} \in I_{T}$. This is shown in the left picture of Figure 2.

Step 3. In other cases, we choose an edge whose start (or end) point is the root of some tree and impose an interior nodal controller to its end (or start) point and an interior point controller at $q$. Under this control strategy, we stabilize the system, shown in the right picture of Figure 2 for instance. 

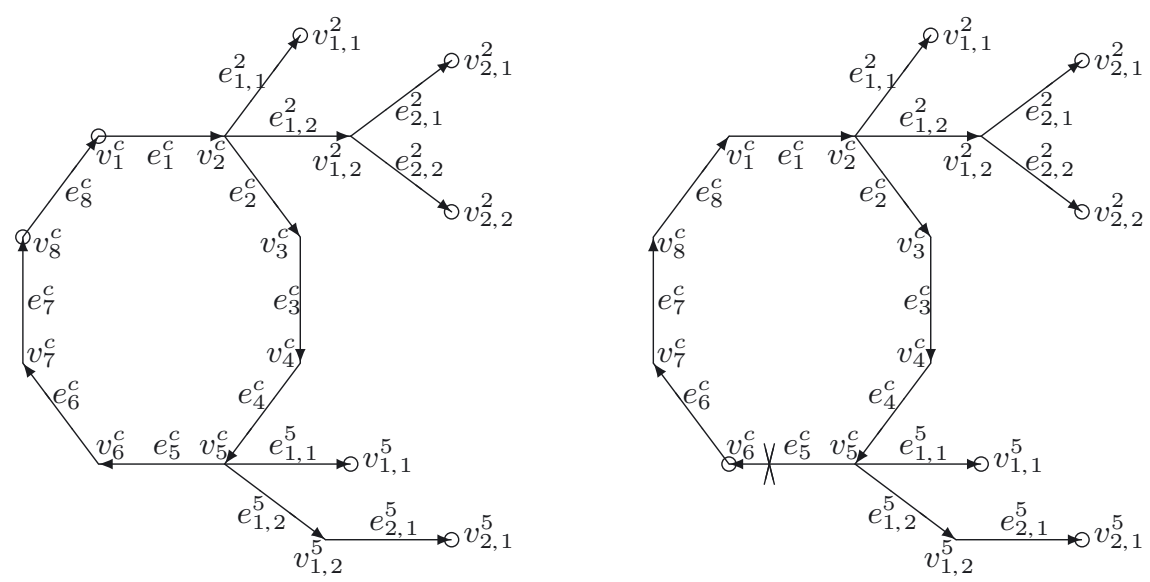

FiguRE 2. Example 1.

Therefore, we have used five boundary controllers and two interior controllers to asymptotically stabilize the wave network expanded on Figure 2. Moreover, no matter the cycle $\mathcal{C}$ satisfies condition (A) or not, we can use two controllers to stabilize asymptotically the system on $\mathcal{C}$, provided that there is at least one tree in the graph $\mathcal{G}$.

\subsection{Example 2}

This example includes a more complex graph that has more trees as shown in Figure 3.

Step 1. Impose the boundary controllers.

Step 2. If there exists an edge pair of the cycle satisfying condition (A), and their start and end points are the roots of some trees (e.g., the edge pairs $\left(e_{1}^{c}, e_{2}^{c}\right)$ or $\left.\left(e_{5}^{c}, e_{6}^{c}\right)\right)$, then there is no need to impose interior controller to asymptotically stabilize the system, shown in the left picture of Figure 3 for instance.

Step 3. In other cases, we choose an edge whose start point and end point are the roots of some trees. We need only one interior point controller at $q$ on this edge to stabilize the system, shown in the right picture of Figure 3 for instance.

\subsection{Example 3}

In this example we consider an extreme case: $\mathcal{G}=\mathcal{C}$ shown as in Figure 4, where three controllers in the cycle $\mathcal{C}$ are really needed.

Obviously, the number of the boundary vertices is 0 (i.e., $N_{B}=0$ ). Therefore, no boundary controller is needed. We need only to consider the interior controller design according to Theorem 3.1.

Step 1. If there is an edge pair, for example $\left(e_{4}^{c}, e_{5}^{c}\right)$, in $\mathcal{G}=\mathcal{C}$ satisfying condition (A), then we add three interior nodal controllers at their start and end points $v_{4}^{c}, v_{5}^{c}$ and $v_{6}^{c}$ to asymptotically stabilize it, shown in the left figure of Figure 4.

Step 2. If $\mathcal{G}=\mathcal{C}$ fails to satisfy condition $(\mathrm{A})$, then we arbitrarily choose an edge $e_{k}^{c}, k \in I_{c}\left(e . g\right.$. $\left.e_{4}^{c}\right)$, and impose an interior point controller at $q$ on $e_{4}^{c}$, and two interior nodal controllers at its start point $v_{4}^{c}$ and end point $v_{5}^{c}$, shown in the right figure of Figure 4.

Remark 4.1. In Example 1, the graph has 15 vertices and 5 boundary vertices. We can asymptotically stabilize the system by at most 7 controllers. In Example 2, the graph has 25 vertices and 12 boundary vertices. We use at most 13 controllers to asymptotically stabilize the system. They need fewer number of controllers than that stated in Theorem 3.1. In Example 3, the graph has no tree-shaped structure. We impose 3 controllers on the cycle $\mathcal{C}$ to asymptotically stabilize the system. Note that $N_{B}=0$ in this example, so the number of the controllers is the same as described in Theorem 3.1. 

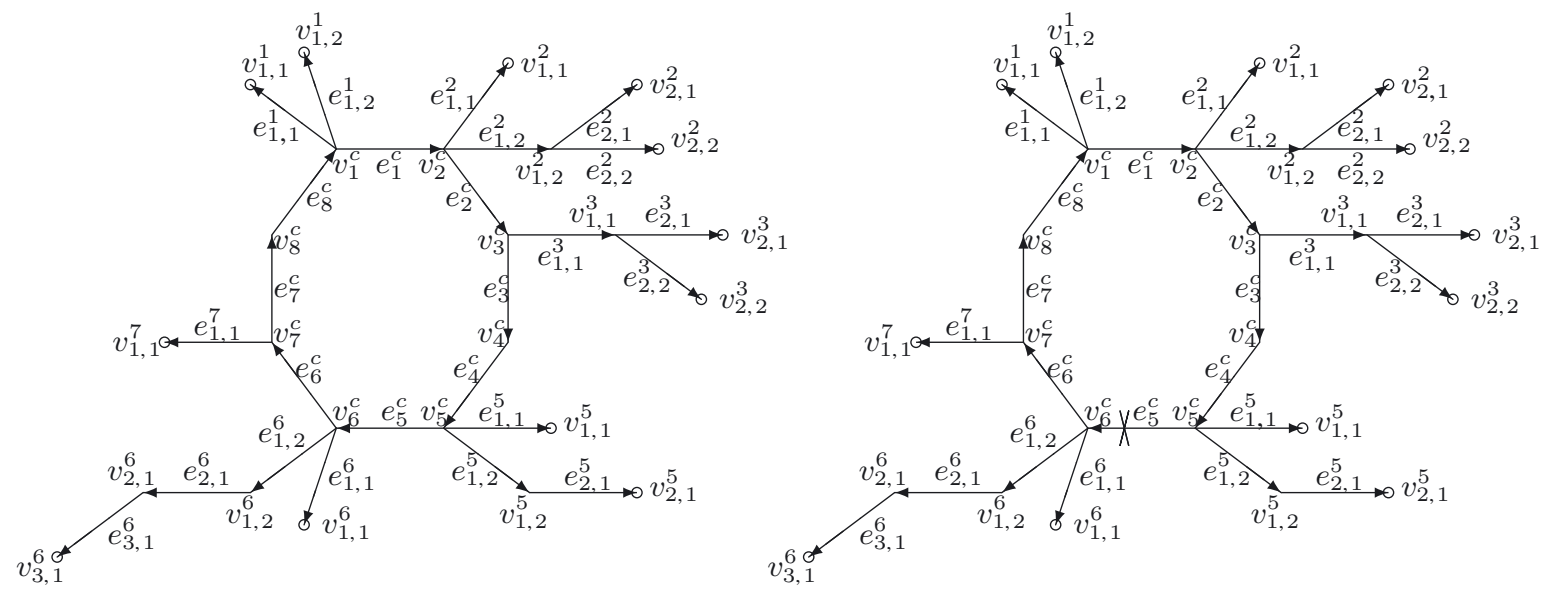

Figure 3. Example 2.
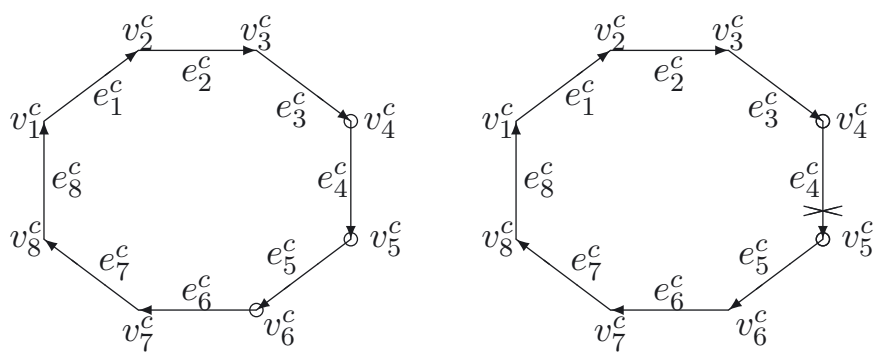

Figure 4. Example 3.

\subsection{Reduction of the boundary controllers}

In preceding examples we concern with reducing the number of interior controllers. In this subsection, we emphasize that for a concrete graph, the number of boundary controllers can also be reduced according to its shape and physical parameters in the wave equation. For instance, if we have

$$
m_{k}^{c}=m_{j, i}^{k}=T_{k}^{c}=T_{j, i}^{k}=1, \quad k \in I_{c}, j=1,2, \ldots, p_{k}, i=1,2, \ldots, N_{k, j}
$$

in Examples 1 and 2, then four controllers can asymptotically stabilize the system in Example 1; and seven controllers can asymptotically stabilize the system in Example 2. The positions of the controllers are shown in Figure 5. The proof of their stability is also carried out by "cutting-edge" method.

As an example, we briefly present the stabilization analysis of the wave network shown in the left figure of Figure 5. It is based on the proof of Theorem 3.1. The procedure of the "cutting-edge" is shown in Figure 6, where the dots denote the vertices at which the displacements are 0 .

Step 1. Since the boundary vertices $v_{2,2}^{2}, v_{2,1}^{5} \in I_{\Gamma}$ are equipped with controllers, we "cut" the edges $e_{2,2}^{2}, e_{2,1}^{5}$ and $e_{1,2}^{5}$. The rest subgraph is $\mathcal{G}_{1}$ shown in the left figure of Figure 6. Now the displacements at vertices $v_{5}^{c}$ and $v_{1,2}^{2}$ are 0 .

Step 2. Since $v_{5}^{c} \in I_{T}$ is a root, $v_{6}^{c}$ is imposed with a controller, and there is an interior point controller at $q$ on edge $e_{5}^{c}$, so we can "cut" edge $e_{5}^{c}$, thus the edges $e_{6}^{c}, e_{7}^{c}, e_{8}^{c}, e_{1}^{c}$ can also be "cut". The rest subgraph is $\mathcal{G}_{2}$ shown in the right figure of Figure 6. Now the displacement at vertex $v_{2}^{c}$ is also 0 . 

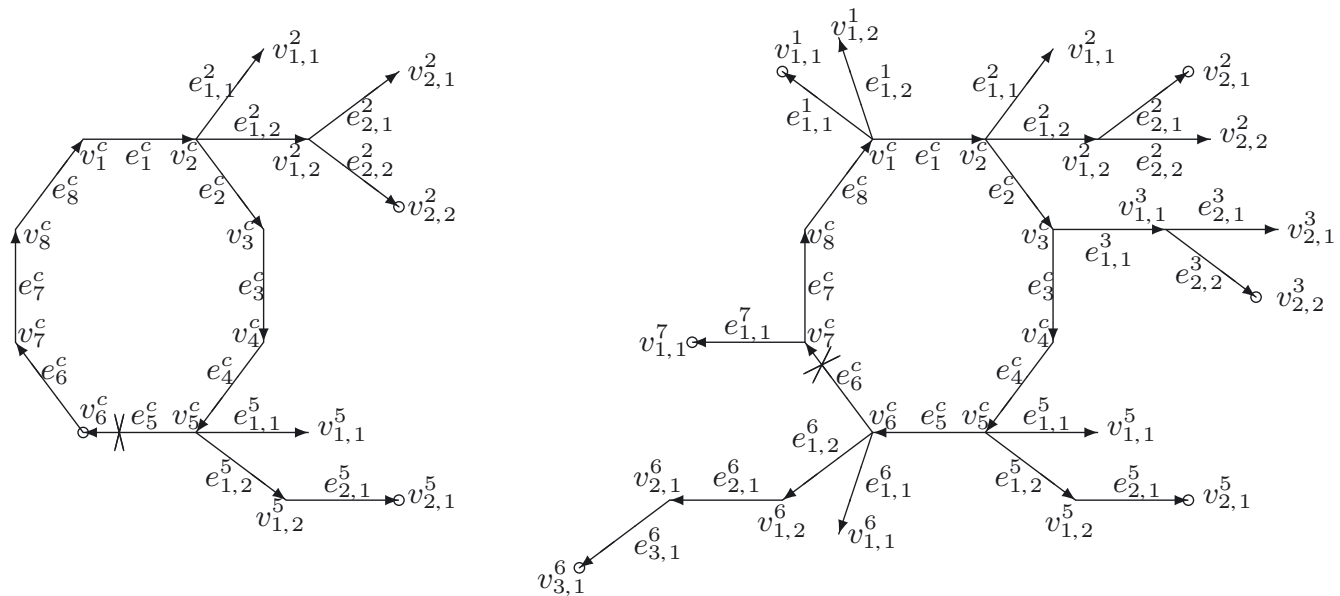

FiguRE 5. Controller design for Examples 1 and 2 when all the parameters are 1.

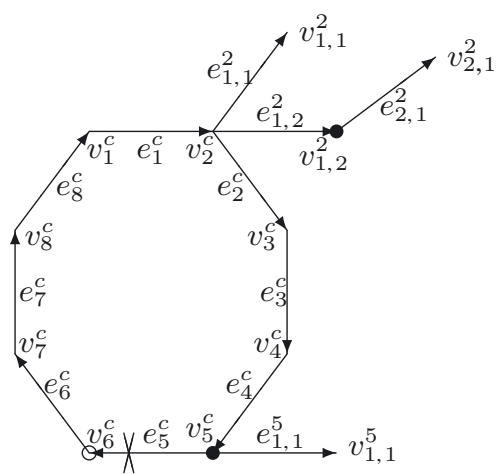

$\mathcal{G}_{1}$.

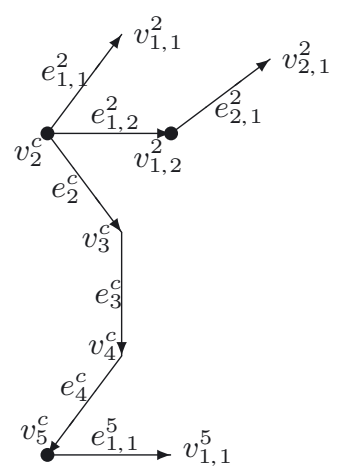

$\mathcal{G}_{2}$.

Figure 6. "Cutting-edge" of Example 1 under the controller design in Figure 5.

Step 3. Now we analyze the eigenvalue problem of the rest subgraph $\mathcal{G}_{2}$ on the imaginary axis under the assumption (4.1). Similar to the proof of Theorem 3.1, we can prove that this eigenvalue problem has only zero solution. So the rest subgraph $\mathcal{G}_{2}$ can be "cut". Hence the wave network in Example 1 is asymptotically stabilized by the control strategy shown in the left figure of Figure 5.

The stabilization analysis of the system shown in the right figure of Figure 5 can be similarly carried out.

\section{Conclusion}

In this paper, we introduce the "cutting-edge" method in the controller design. Using this method, we can get the control strategy which includes the number and locations of the controllers, and the asymptotic stability of the closed form. For bush-type wave networks, we prove theoretically the validity of the "cutting-edge" method. As asserted in Theorem 3.1, at most $N_{B}+3$ controllers can stabilize the bush-type wave networks asymptotically. Moreover, for a concrete graph, we can further reduce the number of controllers according to its shape and physical parameters in the wave equations, as shown in the examples in Section 4. 


\section{A. APPENDIX}

Here we list some definitions and proofs omitted in the previous sections. Some symbols used here may be different from the ones in the previous sections only for simplicity and clarity.

\section{A.1. Notions in graph theory}

Definition A.1 ([5]). Let $G$ be a simple connected and directed graph with vertex set $V=\left\{v_{1}, v_{2}, \ldots, v_{m}\right\}$ and edge set $E=\left\{e_{1}, e_{2}, \ldots, e_{n}\right\}$.

The adjacency matrix of $G$ is the matrix $A=\left(a_{i j}\right)_{m \times m}$, whose entry $a_{i j}=1$ if there is an edge $e \in E$ such that $e=\left[v_{i}, v_{j}\right]$; and $a_{i j}=0$ otherwise. Moreover, if $a_{i j}=1$, we say that $v_{i}$ and $v_{j}$ are adjacent.

The incoming incidence matrix of $G$, denoted by $\Phi^{+}$, is an $m \times n$ matrix whose entry $\phi_{i j}^{+}$is defined by

$$
\phi_{i j}^{+}= \begin{cases}1, & v_{i} \text { is the end point of } e_{j} \\ 0, & \text { otherwise }\end{cases}
$$

and the outgoing incidence matrix of $G$, denoted by $\Phi^{-}$, is also an $m \times n$ matrix whose entry $\phi_{i j}^{-}$is defined by

$$
\phi_{i j}^{-}= \begin{cases}1, & v_{i} \text { is the start point of } e_{j} \\ 0, & \text { otherwise. }\end{cases}
$$

The incidence matrix $\Phi$ is defined by $\Phi:=\Phi^{+}-\Phi^{-}$.

For $v \in V, d^{+}(v)$ denotes the number of the incoming edges at the vertex $v$, which is called the in-degree of $v$, and $d^{-}(v)$ denotes the number of the outgoing edges of $v$, which is called the out-degree at $v$; the number $d(v):=d^{+}(v)+d^{-}(v)$ is said to be the degree of the vertex $v$.

Define the $m \times m$ diagonal matrix $D=\operatorname{diag}\left(d\left(v_{1}\right), d\left(v_{2}\right), \ldots, d\left(v_{m}\right)\right)$. A direct verification shows that [5]

$$
\Phi \Phi^{T}=D-A .
$$

\section{A.2. Proof of $S+\Phi T \Phi^{T}$ being positive definite}

Proposition A.1. Let $G$ be a simple connected and directed graph with vertex set $V=\left\{v_{1}, v_{2}, \ldots, v_{m}\right\}$ and edge set $E=\left\{e_{1}, e_{2}, \ldots, e_{n}\right\}$. Set $n \times n$ diagonal matrix $T=\operatorname{diag}\left(T_{1}, T_{2}, \ldots, T_{n}\right)$ with entries $T_{k}>0, k=1,2, \ldots, n$, and $m \times m$ diagonal matrix $S=\operatorname{diag}\left(\beta_{1}, \beta_{2}, \ldots, \beta_{m}\right)$ with entries $\beta_{k} \geq 0, k=1,2, \ldots, m$ and $\sum_{k=1}^{m} \beta_{k}>0$. Then $S+\Phi T \Phi^{T}$ is a positive definite matrix.

Proof. Note that $T$ is a positive definite matrix, and $S$ is a nonnegative matrix. Then for any $y \in \mathbb{C}^{m}$, we have

$$
(S y, y)_{\mathbb{C}^{m}}+\left(\Phi T \Phi^{T} y, y\right)_{\mathbb{C}^{m}}=(S y, y)_{\mathbb{C}^{m}}+\left(T \Phi^{T} y, \Phi^{T} y\right)_{\mathbb{C}^{n}} \geq 0
$$

which implies that $S+\Phi T \Phi^{T}$ is a nonnegative matrix. To prove that it is a positive definite matrix, we need only to prove that it is a nonsingular matrix.

Let $\Phi=\left(\phi_{i j}\right)_{m \times n}$, a simple calculation gives

$$
\Phi T \Phi^{T}=\left(\sum_{k=1}^{n} \phi_{i k} T_{k} \phi_{j k}\right)_{m \times m}:=\left(T_{i j}\right)_{m \times m}
$$

where

$$
T_{i j}= \begin{cases}0, & v_{i} \text { and } v_{j} \text { are not adjacent } \\ -T_{k}, & v_{i} \text { and } v_{j} \text { are adjacent and } e_{k}=\left[v_{i}, v_{j}\right] \text { or } e_{k}=\left[v_{j}, v_{i}\right] \\ \sum_{k \in I\left(v_{i}\right)} T_{k}, & i=j\end{cases}
$$

where the index set $I\left(v_{i}\right)=\left\{k \mid e_{k}=\left[v_{i}, v_{j}\right]\right.$ or $\left.e_{k}=\left[v_{j}, v_{i}\right], j=1,2, \ldots, m\right\}$. 
Thus (A.1) reads that the sum of the elements of each line (column) of matrix $\Phi T \Phi^{T}$ is zero, i.e.,

$$
\left\{\begin{array}{c}
T_{i i}=-\sum_{j=1, j \neq i}^{m} T_{i j}=-\sum_{j=1, j \neq i}^{m} \sum_{k=1}^{n} \phi_{i k} T_{k} \phi_{k j} \\
T_{i i}=-\sum_{j=1, j \neq i}^{m} T_{j i}=-\sum_{j=1, j \neq i}^{m} \sum_{k=1}^{n} \phi_{j k} T_{k} \phi_{k i}
\end{array}\right.
$$

i.e., $\Phi T \Phi^{T}$ is diagonally dominant.

Without loss of generality we assume that $\beta_{1}>0$ and $\beta_{j}=0, j \neq 1$. Now we calculate the determinant of $S+\Phi T \Phi^{T}:$

$$
\begin{aligned}
\operatorname{det}\left(S+\Phi T \Phi^{T}\right) & =\left|\begin{array}{ccccc}
T_{11}+\beta_{1} & T_{12} & T_{13} & \cdots & T_{1 m} \\
T_{21} & T_{22} & T_{23} & \cdots & T_{2 m} \\
T_{31} & T_{32} & T_{33} & \cdots & T_{3 m} \\
\vdots & \vdots & \cdots & \cdots & \vdots \\
T_{m 1} & T_{m 2} & T_{m 3} & \cdots & T_{m m}
\end{array}\right| \\
& =\left|\begin{array}{ccccc}
\beta_{1} & T_{12} & T_{13} & \cdots & T_{1 m} \\
0 & T_{22} & T_{23} & \cdots & T_{2 m} \\
0 & T_{32} & T_{33} & \cdots & T_{3 m} \\
\vdots & \vdots & \cdots & \cdots & \vdots \\
0 & T_{m 2} & T_{m 3} & \cdots & T_{m m}
\end{array}\right|=\beta_{1}\left|\begin{array}{cccc}
T_{22} & T_{23} & \cdots & T_{2 m} \\
T_{32} & T_{33} & \cdots & T_{3 m} \\
\vdots & \cdots & \cdots & \vdots \\
T_{m 2} & T_{m 3} & \cdots & T_{m m}
\end{array}\right| .
\end{aligned}
$$

Since the matrix

$$
\left[\begin{array}{cccc}
T_{22} & T_{23} & \cdots & T_{2 m} \\
T_{32} & T_{33} & \cdots & T_{3 m} \\
\vdots & \cdots & \cdots & \vdots \\
T_{m 2} & T_{m 3} & \cdots & T_{m m}
\end{array}\right]
$$

is a weakly diagonally dominant, so its determinant is nonzero. Therefore, $\operatorname{det}\left(S+\Phi T \Phi^{T}\right) \neq 0$, i.e., $S+\Phi T \Phi^{T}$ is a positive definite matrix. The proof is then complete.

\section{A.3. The proof of Theorem 1.1}

The proof will be carried out by three steps.

Step 1. The subspace $X_{1}$ is closed and invariant under $T(t)$ and $T^{*}(t)$.

Clearly, $X_{1}$ is a closed subspace of $X$. We now prove that $X_{1}$ is invariant under $T^{*}(t)$, i.e., for any $x \in X_{1}$, we have $T^{*}(s) x \in X_{1}, \forall s \geq 0$.

Since $T(t)$ is a semigroup of contraction, so is $T^{*}(t)$. Thus for any $x \in X$,

$$
\left\|T^{*}(t+s) x\right\|=\left\|T^{*}(t) T^{*}(s) x\right\| \leq\left\|T^{*}(s) x\right\|, \quad \forall t, s \geq 0 .
$$

This implies that $\left\|T^{*}(t) x\right\|$ is a decreasing function in $t$. Hence

$$
z(t)=\left\|T^{*}(t) x\right\|^{2}-\left\|T^{*}(t+s) x\right\|^{2} \rightarrow 0, \quad t \rightarrow \infty .
$$

Note that

$$
\begin{aligned}
z(t) & =\left(T^{*}(t) x, T^{*}(t) x\right)-\left(T^{*}(t+s) x, T^{*}(t+s) x\right) \\
& =\left\|\left[I-T(s) T^{*}(s)\right]^{\frac{1}{2}} T^{*}(t) x\right\|^{2} \rightarrow 0, \quad t \rightarrow \infty
\end{aligned}
$$


where we have used the fact that $I-T(s) T^{*}(s)$ is a self-adjoint and non-negative operator. So we have

$$
\left[I-T(s) T^{*}(s)\right] T^{*}(t) x \rightarrow 0, \quad t \rightarrow \infty .
$$

Hence

$$
T^{*}(s)\left[I-T(s) T^{*}(s)\right] T^{*}(t) x=\left[I-T^{*}(s) T(s)\right] T^{*}(t+s) x \rightarrow 0, \quad t \rightarrow \infty, \quad x \in X .
$$

Therefore, for any $x \in X$ and $s \geq 0$, it holds that

$$
\left[I-T^{*}(s) T(s)\right] T^{*}(t) x \rightarrow 0, \quad t \rightarrow \infty .
$$

For any $x, y \in X$,

$$
\begin{aligned}
& \left(\left[I-T(s) T^{*}(s)\right] T^{*}(t) x, y\right)=\left(x, T(t)\left[I-T(s) T^{*}(s)\right] y\right) \rightarrow 0, \quad t \rightarrow \infty \\
& \left(\left[I-T^{*}(s) T(s)\right] T^{*}(t) x, y\right)=\left(x, T(t)\left[I-T^{*}(s) T(s)\right] y\right) \rightarrow 0, \quad t \rightarrow \infty
\end{aligned}
$$

which implies in the sense of weak convergence that

$$
\text { (weak) } T(t)\left[I-T(s) T^{*}(s)\right] y \rightarrow 0, \quad(\text { weak }) T(t)\left[I-T^{*}(s) T(s)\right] y \rightarrow 0, \quad t \rightarrow \infty .
$$

Now let $x \in X_{1}$, then we have

$$
\text { (weak) } T(t+s) T^{*}(s) x=T(t) x-T(t)\left[I-T(s) T^{*}(s)\right] x \rightarrow 0, \quad t \rightarrow \infty .
$$

For any $x \in X_{1} \cap D\left(A^{*}\right)$, there exists a $y \in X$ such that $x=R^{*}(\lambda, A) y, \lambda \in \rho(A)$. Since $R(\lambda, A)$ is compact, so is $R^{*}(\lambda, A)$. By the property of compact operator, we have, in the sense of norm of $X$, that

$$
T(t+s) T^{*}(s) x=T(t+s) T^{*}(s) R^{*}(\lambda, A) y \rightarrow 0, \quad t \rightarrow \infty .
$$

Noting that $\overline{X_{1} \cap D\left(A^{*}\right)}=X_{1}$, the Uniformly Boundedness Theorem asserts that

$$
T(t+s) T^{*}(s) x \rightarrow 0, \quad t \rightarrow \infty, \quad \forall x \in X_{1} .
$$

Besides, it obviously holds that

$$
\lim _{t \rightarrow \infty}\left\|T^{*}(t) T^{*}(s) x\right\|=\lim _{t \rightarrow \infty}\left\|T^{*}(s+t) x\right\|=0, \quad x \in X_{1} .
$$

Therefore, $T^{*}(s) x \in X_{1}$, i.e., $X_{1}$ is invariant under $T^{*}(t)$. Similarly, we can prove that $X_{1}$ is invariant under $T(t)$.

Step 2. The subspace $X_{2}$ is closed and invariant under $T(t)$ and $T^{*}(t)$.

Since $T(t) T^{*}(t)$ is a self-adjoint operator, so $\|T(t) x\|=\left\|T^{*}(t) x\right\|=\|x\|$ is equivalent to

$$
x=T(t) T^{*}(t) x=T^{*}(t) T(t) x .
$$

Thus we can rewrite $X_{2}$ as

$$
X_{2}=\bigcap_{t \geq 0} \mathcal{N}\left(I-T^{*}(t) T(t)\right) \cap \mathcal{N}\left(I-T(t) T^{*}(t)\right) .
$$

This indicates that $X_{2}$ is a closed subspace of $X$. Moreover, from (A.4) we can also see that the restriction of $T(t)$ on $X_{2}$ can be embedded in a $C_{0}$ group [18]. Therefore, $X_{2}$ is invariant under $T(t)$ and $T^{*}(t)$.

Step 3. The subspaces $X_{1}$ and $X_{2}$ are orthogonal, and $X=X_{1} \oplus X_{2}$. 
Since $X$ is a Hilbert space and $X_{1}$ is a closed subspace of $X$, then we have $X=X_{1} \oplus X_{1}^{\perp}$. Firstly, we shall prove that $X_{1}^{\perp} \subset X_{2}$. Indeed, let $\lambda>0$, since $R(\lambda, A)$ is compact, then we have from (A.3) that

$$
T(t) R(\lambda, A)\left[I-T(s) T^{*}(s)\right] x \rightarrow 0, \quad T(t) R(\lambda, A)\left[I-T^{*}(s) T(s)\right] x \rightarrow 0, \quad t \rightarrow \infty
$$

which implies that $R(\lambda, A)\left[I-T(s) T^{*}(s)\right] x \in X_{1}$ and $R(\lambda, A)\left[I-T^{*}(s) T(s)\right] x \in X_{1}$. Thus

$$
\begin{aligned}
& {\left[I-T(s) T^{*}(s)\right] x=\lim _{\lambda \rightarrow+\infty} \lambda R(\lambda, A)\left[I-T(s) T^{*}(s)\right] x \in X_{1},} \\
& {\left[I-T^{*}(s) T(s)\right] x=\lim _{\lambda \rightarrow+\infty} \lambda R(\lambda, A)\left[I-T^{*}(s) T(s)\right] x \in X_{1} .}
\end{aligned}
$$

In particular, for any $y \in X_{1}^{\perp},\left[I-T^{*}(s) T(s)\right] y \in X_{1}$ and $\left[I-T(s) T^{*}(s)\right] y \in X_{1}$. Hence

$$
0=\left(\left[I-T^{*}(s) T(s)\right] y, y\right)=\|y\|^{2}-\|T(s) y\|^{2}, \quad 0=\left(\left[I-T(s) T^{*}(s)\right] y, y\right)=\|y\|^{2}-\left\|T^{*}(s) y\right\|^{2} .
$$

That is

i.e., $X_{1}^{\perp} \subset X_{2}$.

$$
\left\|T^{*}(s) y\right\|=\|T(s) y\|=\|y\|, \quad y \in X_{1}^{\perp}, \quad s \geq 0
$$

Now we prove $X_{2} \subset X_{1}^{\perp}$. From the definition of $X_{2}$, we get

$$
\left(\left[I-T^{*}(s) T(s)\right] y, y\right)=0, \quad\left(\left[I-T(s) T^{*}(s)\right] y, y\right)=0, \quad y \in X_{2}
$$

which implies that $\left[I-T^{*}(s) T(s)\right] y=\left[I-T(s) T^{*}(s)\right] y=0$ since $I-T(s) T^{*}(s)$ is self-adjoint.

Thus for any $x \in X_{1}$ and $y \in X_{2}$, we have

$$
\left(\left[I-T^{*}(s) T(s)\right] x, y\right)=0, \quad\left(x,\left[I-T(s) T^{*}(s)\right] y\right)=0
$$

and hence

$$
|(x, y)|=\left|\left(T^{*}(s) T(s) x, y\right)\right|=|(T(s) x, T(s) y)| \leq|| T(s) x|||| T(s) y\|=\| T(s) x|||| y|| \rightarrow 0, \quad s \rightarrow \infty
$$

i.e., $(x, y)=0$. So $y \in X_{1}^{\perp}$, hence $X_{2} \subset X_{1}^{\perp}$. Therefore, $X_{2}=X_{1}^{\perp}$. The proof is then complete.

\section{REFERENCES}

[1] K. Ammari and M. Jellouli, Stabilization of star-shaped tree of elastic strings. Differential Integral Equations 17 (2004) $1395-1410$

[2] K. Ammari and M. Jellouli, Remark on stabilization of tree-shaped networks of strings. Appl. Math. 52 (2007) $327-343$.

[3] K. Ammari and S. Nicaise, Polynomial and analytic stabilization of a wave equation coupled with a Euler-Bernoulli beam. Math. Methods Appl. Sci. 32 (2009) 556-576.

[4] K. Ammari, M. Jellouli and M. Khenissi, Stabilization of generic trees of strings. J. Dyn. Control Syst. 11 (2005) 177-193.

[5] J.A. Bondy and U.S.R. Murty, Graph Theory, Graduate Texts in Mathematics Series. Springer-Verlag, New York (2008).

[6] R. Dáger, Observation and control of vibrations in tree-shaped networks of strings. SIAM J. Control Optim. 43 (2004) $590-623$.

[7] R. Dáger and E. Zuazua, Controllability of star-shaped networks of strings. C. R. Acad. Sci. Paris, Sér. I 332 (2001) $621-626$.

[8] R. Dáger and E. Zuazua, Controllability of tree-shaped networks of vibrating strings. C. R. Acad. Sci. Paris, Sér. I 332 (2001) $1087-1092$.

[9] R. Dáger and E. Zuazua, Wave propagation, observation and control in 1-d flexible multistructures, Mathématiques and Applications 50. Springer-Verlag, Berlin (2006).

[10] M. Gugat, Boundary feedback stabilization by time delay for one-dimensional wave equations. IMA J. Math. Control Inform. 27 (2010) 189-204.

[11] B.Z. Guo and Z.C. Shao, On exponential stability of a semilinear wave equation with variable coefficients under the nonlinear boundary feedback. Nonlinear Anal. 71 (2009) 5961-5978.

[12] D. Jungnickel, Graphs, Networks and Algorithms, Algorithms and Computation in Mathematics 5. Springer-Verlag, New York, third edition (2008). 
[13] J.E. Lagnese, G. Leugering and E.J.P.G. Schmidt, Modeling, analysis and control of dynamic elastic multi-link structures Systems and control: Foundations and applications. Birkhäuser-Basel (1994).

[14] G. Leugering and E.J.P.G. Schmidt, On the control of networks of vibrating strings and beams. Proc. of the 28th IEEE Conference on Decision and Control 3 (1989) 2287-2290.

[15] G. Leugering and E. Zuazua, On exact controllability of generic trees. ESAIM: Proc. 8 (2000) 95-105.

[16] Yu.I. Lyubich and V.Q. Phóng, Asymptotic stability of linear differential equations in Banach spaces. Studia Math. 88 (1988) $34-37$.

[17] S. Nicaise and J. Valein, Stabilization of the wave equation on 1-d networks with a delay term in the nodal feedbacks. Netw. Heterog. Media 2 (2007) 425-479.

[18] A. Pazy, Semigroups of linear operators and applications to partial differential equations. Springer-Verlag, Berlin (1983).

[19] J. Valein and E. Zuazua, Stabilization of the wave equation on 1-d networks. SIAM J. Control Optim. 48 (2009) 2771-2797.

[20] G.Q. Xu, D.Y. Liu and Y.Q. Liu, Abstract second order hyperbolic system and applications to controlled network of strings. SIAM J. Control Optim. 47 (2008) 1762-1784. 